\title{
A structural model for microtubule minus-end recognition and protection by CAMSAP proteins
}

\author{
Joseph Atherton $\# 1$, Kai Jiang ${ }^{\# 2}$, Marcel M. Stangier ${ }^{3}$, Yanzhang Luo ${ }^{4}$, Shasha Hua ${ }^{2}$, \\ Klaartje Houben ${ }^{4}$, Jolien J.E. van Hooff ${ }^{5,6,7}$, Agnel-Praveen Joseph ${ }^{1}$, Guido Scarabelli ${ }^{8}$, \\ Barry J. Grant ${ }^{8}$, Anthony J. Roberts ${ }^{1}$, Maya Topf ${ }^{1}$, Michel O. Steinmetz ${ }^{3,9}$, Marc Baldus ${ }^{4}$, \\ Carolyn A. Moores ${ }^{1, \#}$, and Anna Akhmanova ${ }^{2, \#}$
}

${ }^{1}$ Institute of Structural and Molecular Biology, Birkbeck, University of London, London, United Kingdom ${ }^{2}$ Cell Biology, Department of Biology, Faculty of Science, Utrecht University, Utrecht, the Netherlands ${ }^{3}$ Laboratory of Biomolecular Research, Division of Biology and Chemistry, Paul Scherrer Institut, Villigen PSI, Switzerland ${ }^{4}$ NMR Spectroscopy, Bijvoet Center for Biomolecular Research, Utrecht University, Utrecht, the Netherlands ${ }^{5}$ Hubrecht Institute, Utrecht, the Netherlands ${ }^{6}$ Theoretical Biology and Bioinformatics, Department of Biology, Faculty of Science, Utrecht University, Utrecht, The Netherlands ${ }^{7}$ Molecular Cancer Research, University Medical Center Utrecht, Utrecht, the Netherlands ${ }^{8}$ Department of Computational Medicine and Bioinformatics, University of Michigan Medical School, Ann Arbor, Michigan, USA. ${ }^{9}$ University of Basel, Biozentrum, Basel, Switzerland

\# These authors contributed equally to this work.

\begin{abstract}
CAMSAP and Patronin family members regulate microtubule minus-end stability and localization and thus organize non-centrosomal microtubule networks, which are essential for cell division, polarization and differentiation. Here, we show that the C-terminal CKK domain of CAMSAPs is widely present among eukaryotes and autonomously recognizes microtubule minus ends. Through a combination of structural approaches, we uncover how mammalian CKK binds between two tubulin dimers at the inter-protofilament interface on the outer microtubule surface. In vitro reconstitution assays combined with high resolution fluorescence microscopy and cryo-electron tomography suggest that CKK preferentially associates with the transition zone between curved protofilaments and the regular microtubule lattice. We propose that minus-end-specific features of the inter-protofilament interface at this site form the basis for CKK's minus-end preference. The steric clash between microtubule-bound CKK and kinesin motors explains how CKK protects
\end{abstract}

\footnotetext{
Users may view, print, copy, and download text and data-mine the content in such documents, for the purposes of academic research, subject always to the full Conditions of use:http://www.nature.com/authors/editorial_policies/license.html\#terms

${ }^{\#}$ Corresponding authors: Carolyn A. Moores, c.moores@mail.cryst.bbk.ac.uk; Anna Akhmanova, a.akhmanova@uu.nl. Author contributions

J.A, K.J, M.O.S., M.B., C.A.M. and A.A. designed experiments, analyzed data and wrote the paper. C.A.M and A.A. coordinated the project. J.A. performed and analysed cryo-EM and cryo-ET experiments, K.J and S.H performed in vitro reconstitution experiments, M.M.S. performed X-ray crystallography and biophysical experiments, Y.L and K.H. performed and analysed NMR experiments, J. van H. performed bioinformatics analysis, A.-P. J, M.T performed cryo-EM based molecular modelling, G.S. and B.J.G performed molecular dynamics calculations and A.J.R performed subtilisin-MT TIRFM assay.

The authors have no competing financial interests.
} 
microtubule minus ends against kinesin-13-induced depolymerization and thus controls the stability of free microtubule minus ends.

\section{Introduction}

Microtubules (MTs) are highly dynamic polymers, which assemble and disassemble from their two ends, the fast-growing plus end and the slow-growing minus end. While many data are available about the regulation of MT plus ends ${ }^{1,2}$, much less is known about the proteins specifically regulating MT minus ends, despite the fact that MT minus end organization defines the architecture of cellular MT arrays ${ }^{3,4}$. The best-studied MT nucleating and minus-end binding factor is the $\gamma$-tubulin ring complex $(\gamma \text {-TuRC })^{5}$. Recently, the members of calmodulin-regulated spectrin-associated protein (CAMSAP) and Patronin family were shown to control non-centrosomal MT minus-end organization independently of $\gamma$-TuRC in different systems, including the mitotic spindle in insect cells ${ }^{6-8}$, cortically attached MT arrays in epithelial cells ${ }^{9-13}$ and MT bundles in neurons ${ }^{14-17}$.

CAMSAP1, CAMSAP2 and CAMSAP3 (in vertebrates) and Patronin (in invertebrates) recognize and track uncapped, growing MT minus ends ${ }^{18,19}$. In mammals, CAMSAP2 and CAMSAP3 are deposited on MT lattices formed by MT minus-end polymerization and in this way generate stable MT stretches that can serve as a source of non-centrosomal MT outgrowth $^{18,20}$. In contrast, CAMSAP1 tracks growing MT minus ends but does not decorate them ${ }^{18}$. CAMSAPs and Patronin contain a C-terminal CKK domain (C-terminal domain common to CAMSAP1, KIAA1078 and KIAA1543) as well as an N-terminal Calponin Homology $(\mathrm{CH})$ domain and several coiled-coil regions ${ }^{21}$. In mammalian CAMSAPs, the minus-end recognition was shown to depend on the CKK domain, while the ability of CAMSAP2 and CAMSAP3 to stay attached to the MT lattice was associated with additional adjacent regions ${ }^{18}$. However, in fly Patronin, the minus-end recognition was proposed to be mediated by the unstructured linker region in combination with the adjacent C-terminal coiled coil ${ }^{19}$. The deletion of the CKK domain in CAMSAP3 causes a loss-offunction phenotype in mice ${ }^{10}$, and in worms, the CKK domain of Patronin (PTRN-1) was necessary and sufficient for supporting proper MT dynamics and axon regeneration ${ }^{17}$, demonstrating the functional importance of this domain.

Here, we set out to investigate the nature of MT minus-end specificity of CAMSAPs. Structural analysis showed that the globular CKK domain binds to a unique site between two tubulin dimers at the inter-protofilament interface. Fluorescence microscopy demonstrated that the high-affinity site for CKK binding is located a few tubulin dimers behind the outmost MT minus end. Cryo-electron tomography of MT minus ends revealed a heterogeneous array of gently curved protofilaments that retain lateral interactions. Based on these data, we propose that the CKK preferentially binds to the transition zone between the regular lattice and the curved sheet-like structure of the minus-end extremity, which presents a subtly altered inter-protofilament interface that is optimal for CKK binding. Finally, our structural and in vitro reconstitution data show that CKK sterically hinders the interaction of kinesin-13 with MT minus ends, explaining how CAMSAPs and Patronin protect MT minus ends against depolymerization by these factors. 


\section{Results}

\section{Minus-end recognition by CAMSAPs depends on the CKK domain, which is conserved in eukaryotes}

We have previously shown that the CKK domain in mammalian CAMSAPs could bind minus ends ${ }^{18}$. To investigate whether other CAMSAP domains could also control minus-end recognition, we have tested the ability of different purified GFP-tagged CAMSAP1 and CAMSAP3 domains to bind to MT minus ends and lattices in vitro. None of the CAMSAP fragments which lacked the CKK domain showed any minus-end preference (Fig. 1a-f and Supplementary Fig. 1a-c). For CAMSAP1, we found that the predicted helical domain and the linker which precede the CKK had only very weak MT affinity, but increased MT binding, though not minus end selectivity of CAMSAP1 CKK (Fig.1a-f). An additional negatively charged linker region of CAMSAP1 (Supplementary Fig. 1d), located upstream of its third coiled coil domain, suppressed MT lattice binding and enhanced minus end selectivity when present together with the CKK domain (Fig.1a-e; this construct was termed CAMSAP $1_{\text {mini }}$ ). For CAMSAP3, we confirmed that the MT binding domain located between coiled coils 2 and 3 specifically binds to GMPCPP but not GDP MTs, as we have demonstrated previously ${ }^{18}$ (Supplementary Fig. 1a-c). The predicted helical domain of CAMSAP3 together with the adjacent linker could also specifically bind to GMPCPP MTs without showing end preference (Supplementary Fig. 1a-c). We conclude that the minus-end preference of CAMSAPs depends on the CKK domains, while additional domains modulate their end selectivity or the ability to decorate MT lattice.

To establish whether CKK-mediated minus-end binding defines an evolutionarily conserved mechanism, we screened a set of nearly a hundred eukaryotic genomes and found that homologs of the CKK domain are found in all eukaryotic supergroups, except for Amoebozoa, although they were absent in some well-studied lineages such as dikaryan fungi and land plants (Supplementary Fig. 2 and Supplementary Table 1). Parsimoniously interpreting the occurrences of CKK across eukaryotes, we conclude that this domain was likely already present in the genome of the Last Eukaryotic Common Ancestor (LECA), and did not emerge during early animal evolution, as suggested previously ${ }^{21}$. In particular, our discovery of genes encoding CKK-containing proteins in the genomes of various members of the Excavata, a group some consider to be sister to all other eukaryotes (discussed in ${ }^{22}$ ), strongly supports an ancient origin of CKK. While in vertebrates the array of CKKcontaining proteins was expanded by gene duplications, leading to the three CAMSAP paralogs in mammals, this was not the case in many other genomes (see Supplementary Sequence File), suggesting that in most eukaryotes a single copy of the domain fulfils CKK functions.

To test whether the sequence conservation of CKK translates into functional conservation, we next purified GFP-tagged CKK domains derived from fly and worm (Drosophila melanogaster and Caenorhabditis elegans), as well as from four more evolutionarily distant eukaryotes (Trichomonas vaginalis, Tetrahymena thermophila, Naegleria gruberi and Phytophthora infestans). From these six CKK domains tested, four (D. melanogaster, $C$. elegans, T. vaginalis, T. thermophila) specifically tracked the growing minus ends of MTs 
assembled from pig brain tubulin in in vitro MT dynamics reconstitution experiments (Fig. $1 \mathrm{~g}$ ), while the two others ( $N$. gruberi and P. infestans) showed strong binding along the whole MT lattice (data not shown). These data demonstrate that CKK represents a protein module that recognizes MT minus ends, a property that is preserved over long evolutionary distances.

\section{CKK domains bind to an intra-dimer site between protofilaments}

We crystallized and solved the core structure of the mouse CAMSAP3 CKK domain (residues $1121-1239$; denoted $\mathrm{CKK}_{\text {core }}$ ) by X-ray crystallography at a resolution of $1.4 \AA$ (Table 1). Similar to a previously deposited but unpublished NMR structure (PDB ID $1 \mathrm{UGJ}$ ), the $\mathrm{CKK} 3_{\text {core }}$ has a compact, globular structure composed of two $\mathrm{N}$-terminal ahelices, which are connected by a disordered loop (loop1) and pack against a central, fivestranded $\beta$-sheet (Supplementary Fig. 3).

Although CKK shows clear preference for MT minus ends, at high concentrations it can also decorate the whole MT lattice. We reasoned that a high-resolution structure of the latticebound CKK domain might shed light on the mechanism of its MT minus-end recognition. Therefore, we analyzed extended CKK domains of CAMSAP1 and 3 (residues 1474-1613 and 1112-1252, respectively) bound to 13 protofilament (pf) taxol-stabilized MTs by cryoEM. Filtered images of these MTs showed extra density corresponding to CKK domains spaced by $8 \mathrm{~nm}$ (Fig. 2a) indicative of binding every tubulin dimer. A striking feature of these CKK-decorated MTs is the presence of a right-handed skew in the protofilaments. This is evident from both the filtered images and the alignment parameters of the MT segments used for reconstruction, and is specific to the CKK-MT data sets (Fig. 2a,b and Supplementary Fig. 4a). This indicates that CKK binding modifies the MT lattice with which it interacts, consistent with the idea that the regular MT lattice conformation is not the most favored substrate for CKK domains.

Asymmetric 3D reconstructions ( $~ 12$ and $\sim 9 \AA$ resolution of CAMSAP1 and CAMSAP3, respectively) show the CKK domains binding the MT lattice every $8 \mathrm{~nm}$ between protofilaments except at the seam (Fig. 2c and Supplementary Fig. 4b). Using the pseudosymmetry of the MTs, we obtained averaged reconstructions with overall final resolutions that allowed $\alpha$ - and $\beta$-tubulin to be clearly distinguished (FSCtrue 0.143 criteria, ${ }^{23}$ of $5.3 \AA$ (CAMSAP3) and 8̊̊ CAMSAP1) (Fig. 2d,e and Supplementary Fig. 4c-e, Table 2). From these data, we can conclude that CKK domains bind at the tubulin intra-dimer B-lattice interface where conserved differences between $\alpha$ - and $\beta$-tubulin explain this binding site selection (Fig. 3a). The binding site of CKK is distinct from other proteins known to bind between protofilaments, EBs and Doublecortin (DCX), which bind at the vertex of four tubulin dimers and not at the intra-dimer interface (Supplementary Fig. 4f-h). However, some overlap is found with the binding site of kinesin (Supplementary Fig. 4i).

For ease of description, the four tubulin subunits contacting a single CKK domain are referred to as $\beta 1$-, $\beta 2-$, a 1- and a 2-tubulin (see Fig. 2d,e and 3a). In general, the CKK wedges snugly between, and forms more extensive contacts with, the $\beta$-tubulins than with the a-tubulins (Fig. 3a). Contacts between the CKK domain and its MT binding site are distributed across the domain and also involve the N- and C-termini (Fig. $2 \mathrm{~d}$ and 3a,b). To 
confirm and extend the cryo-EM data, we also analyzed the structure of the CKK domain of CAMSAP3 bound to MTs using solid-state NMR (ssNMR), which provides a sensitive means to study ligand and protein binding to MTs at the atomic level ${ }^{24,25}$. To do this, we compared the solution NMR (PDB ID 1UGJ) and our X-ray crystallographic results of free CKK with 2D ssNMR data recorded on $\left[{ }^{13} \mathrm{C},{ }^{15} \mathrm{~N}\right]$ labelled CKK in complex with MTs. This allowed us to identify CKK residues that underwent significant chemical-shift/intensity changes on MT binding (Fig. 3c and Supplementary Fig. 5a-d, labelled red) and those that experienced no significant change (labelled blue). For example, in our EM density, helix-a 1 of the CKK domain sits across the $\beta 1$ - and $\beta 2$-tubulins, while beneath it, loop7 and adjacent regions of the beta barrel wedge in between the $\beta$-tubulin subunits (Fig. 3a-c and Supplementary Fig. 5e,f). Consistent with this, residues from MT-bound CKK helix-a 1 and adjacent loop7 display ssNMR chemical shifts relative to free CKK. Similarly, our cryo-EM and ssNMR data corroborate interactions between CKK loop1 and $\beta 1$-tubulin, CKK loop7 and $\beta 2$-tubulin, while CKK loop3 lies at the intra-dimer interface contacting both $\beta 1$-tubulin and a1-tubulin. CKK loop8 is well positioned to form contacts with a2-tubulin, and our ssNMR data support the involvement of this loop in the MT interaction. However, loop8 is only visible at less conservative cryo-EM density thresholds (Supplementary Fig. 5f) suggesting higher flexibility than the rest of the CKK domain in this region, consistent with its B-factor in the crystal structure (data not shown). Taken together, residues identified as undergoing changes in ssNMR signals were found to cluster on the CKK MT-facing surface. In contrast, residues displaying unaltered ssNMR signals were found to be predominantly solvent-exposed (Fig. 3c and Supplementary Fig. 5b-d). The ssNMR data thus support the EM-derived binding mode at an atomic level.

Neither the CKK N- and C-termini are visible in the crystal structure and both are flexible in solution (PDB ID 1UGJ). However, our cryo-EM and ssNMR data also support the role of the CKK N- and C-termini in MT binding. Density corresponding to the final portion of the $\mathrm{N}$-terminus leading into helix-a1 (starting at S1120) is visible in our cryo-EM reconstruction contacting a 2-tubulin (Fig. 2d, 3a,d), while additional cryo-EM density suggests that more $\mathrm{N}$-terminal regions make additional contacts with $\beta 2$-tubulin (red asterisks, Fig. 3a; red dashed line in Fig. 3d,). The CKK N-terminus is therefore partially stabilized in complex with the MT, consistent with $\mathrm{N}$-terminal truncation construct having lower MT affinity (Fig. 3e). Furthermore, although not readily modelled, cryo-EM density corresponding to the conserved, basic C-terminus of the CKK domain and/or the $\beta$-tubulin C-terminal tail is visible (Fig. 3f). ssNMR measurements in the CAMSAP3 CKK-MT complex suggest that the CKK C-terminus retains some conformational variability when interacting with MTs (Fig. 3c). The location of the CKK C-terminus suggests that it interacts with the flexible acidic $\mathrm{C}$-terminal tail of $\beta 1$-tubulin; this is supported by the observation that the interaction of GFP-tagged CAMSAP3-CKK domain with MTs was significantly reduced when the acidic C-terminal tails of tubulin had been cleaved (Fig. 3g).

We also used molecular dynamics (MD) based energy calculations, which can provide an estimate of the relative energetic contributions of individual residues to complex formation ${ }^{26}$, to analyse the CKK-MT interaction. This analysis also supports the involvement of the regions described above in the CKK-tubulin interface (Supplementary Fig. 6a). Overall analysis of the electrostatic potential of the contact surfaces shows 
complementary charges between the CKK domain and the MT (Supplementary Fig. 6b,c). This is consistent with the large electrostatic contribution to binding energy from MD and the established sensitivity of CAMSAP-MT interaction to ionic strength ${ }^{18,19}$

(Supplementary Fig. 6a,d). CKK residues at the interface with the MT - especially basic ones - are particularly conserved in CKK domains throughout evolution (Supplementary Fig. $2 \mathrm{~b}$ and $6 \mathrm{c}, \mathrm{e})$. In addition, these calculations also highlighted the greater energetic contribution of CKK binding to $\beta$-tubulin over a-tubulin (with a calculated $\Delta \mathrm{G}$ of $-25.1 \pm 5.2$ $\mathrm{kcal} / \mathrm{mol}$ for $\beta$-tubulin positions compared to $-3.6 \pm 5.0 \mathrm{kcal} / \mathrm{mol}$ for $\mathrm{a}$-tubulin). Taken together, our cryo-EM, ssNMR and MD data show that CKK binds through multiple binding sites across two tubulin dimers at their intra-dimer interface between protofilaments, and associates more closely with $\beta$-tubulin.

\section{Analysis of CKK mutants supports the identified CKK-MT interface}

To further validate the identified CKK-MT binding mode, we made a series of CKK mutants in the context of a short version of CAMSAP1 (CAMSAP $1_{\text {mini }}$, residues 1227-1613), which displays a more robust minus-end tracking activity than the CKK domain alone (Fig. 1a-e). Mutations of several conserved positively charged residues reduced the overall binding affinity of CAMSAP $1_{\text {mini }}$ to MTs and their minus ends (Fig. 4a-c), although they did not affect the integrity and folding of the CKK domain, as determined by measuring circular dichroism (CD) spectra and thermal unfolding profiles in the CAMSAP3 CKK context (Supplementary Fig. 7a). Truncation of the N-terminal extension or mutations of positively charged residues in this sequence had a similar effect, as well as truncations of the positively charged C-terminal tail region (Fig. 4a). These data support the validity of our CKK-MT lattice reconstruction and its relevance for CKK binding to the MT minus end.

Surprisingly, a mutation of a conserved asparagine residue (N1492 of CAMSAP1 and N1130 in CAMSAP3) to alanine, which did not affect the integrity of the domain (Supplementary Fig. 7a), dramatically increased the ability of CKK to bind to the MT lattice and reduced, though did not abolish, its selectivity for minus ends (Fig. 4a-c, Supplementary Figure 7b-f). The importance of CAMSAP1 N1492 residue was further emphasized by the observation that mutating it to bulky charged or uncharged amino acids reduced the affinity of CKK for both MT minus ends and lattices, while substituting it for either serine or threonine enhanced MT lattice binding and decreased minus end selectivity even further (Fig. 4d and Supplementary Fig. 7d-f). CAMSAP1 N1492 is expected to contact the Cterminus of helix $\mathrm{H} 4$ of $\beta 2$-tubulin (cyan in Fig. 4b), and our data suggest that the formation of the CKK-MT complex is very sensitive to both to the size and the chemical nature of the side chain in this position. For example, the potential hydrogen bond formation between this residue and $\beta$-tubulin could affect the affinity of the CKK-MT interaction.

We also mutated to alanine several residues close to N1492 in the CKK structure; several of these substitutions had no effect (Supplementary Fig. 7g,h), while the D1572A mutation also enhanced CKK interaction with MT lattice and reduced its minus end selectivity (Fig. 4a). Overall, these experiments identified two main classes of mutations: 1) mutations of positively charged CKK residues, which weakened the interaction between CKK and negatively charged surface of the MT ends and lattice, including less structurally ordered 
regions of both the CKK and MT; 2) mutations that specifically boosted lattice binding. Remarkably, these latter mutations were rather subtle, suggesting that the mechanism of minus end discrimination itself is also subtle.

We reasoned that understanding the mechanism underlying the improved affinity of the N1492A mutant for MTs would provide insight into CKK's minus-end specificity. Therefore, we obtained a 3D reconstruction of the CAMSAP1 CKK N1492A mutant using cryo-EM (Fig. 4e, Supplementary Fig. 8a-d, Table 2). As with the wild type CKK, the N1492A mutant also binds at the tubulin intra-dimer B-lattice interface with the same overall orientation as the wild type CKK (Supplementary Fig. 8a,c,d). However, two distinct structural properties were observed for the mutant: i) less protofilament skew compared to wild type in CKK N1492A decorated MTs (Supplementary Fig.8e) and ii) CKK N1492A itself is subtly shifted towards the main MT body compared to wild type (Fig. 4e). This result suggests that very small changes in the CKK-MT interaction determine binding selectivity of CKK between the lattice and the minus end. It also suggests that the adjustment in the CKK-MT interaction by the N1492A mutant - movement of the CKK domain deeper between protofilaments - could reflect aspects of the mechanism of end selectivity by wild type CKK via selection of a tubulin conformation only present at MT minus ends.

We also attempted to polymerize tubulin in the presence of a high concentration of CKK. In the presence of the wild type CKK, only very few short microtubules formed, and instead a variety of tubulin oligomers were generated that formed clumps. In contrast, in the presence of saturating concentrations of the N1492A mutant, many longer, predominantly 13 protofilament microtubules formed (Supplementary Figure 8f). These data support the idea that the wild type CKK preferentially interacts with a polymeric conformation of tubulin that is distinct from the normal MT structure, while the binding of the N1492A mutant is more compatible with the regular MT lattice.

\section{The high affinity CKK binding site at MT minus ends is insensitive to the tubulin nucleotide state and lies a few tubulin dimers behind the outmost minus end}

Having defined the CKK-MT binding mode, we set out to determine whether the state of MT minus ends affects CKK binding. We found that the binding of CAMSAP1 $1_{\text {mini }}$ to MT minus ends was insensitive to the nucleotide state of MTs, as the protein bound well to the minus ends of GMPCPP-stabilized (GTP like) or taxol-stabilized (GDP) MTs, as well as dynamic GTP or GTP $\gamma$ S (GDP-Pi like) MTs (Fig. 5a,b). In contrast, we observed no accumulation of CAMSAP1 $1_{\text {mini }}$ at depolymerizing MT minus ends (Fig. 5c), suggesting that their structure does not support CKK binding.

To determine the precise localization of CKK on MT minus ends, the model-convolution method $^{27,28}$ was implemented to determine the relative positions of the CKK and the MT minus end from their one dimensional intensity profiles along the MT axis (Fig. 5d,e). Our data showed that CKK accumulates $\sim 16 \mathrm{~nm}$ - equivalent to a few tubulin dimers - behind the outmost MT minus end (estimated measurement error: $\sim 8 \mathrm{~nm}$; see Methods). This result suggests that some structural features of MT minus ends at this specific location form a preferred CKK binding site. 


\section{Cryo-electron tomography shows that inter-protofilament contacts are preserved at MT ends}

To get insight into the structure of this binding site, we analyzed individual ends of GMPCPP-stabilized MTs by cryo-electron tomography (Fig. 5f). Because no averaging was imposed, the tomographic 3D reconstructions contain information about overall polymer configuration and thus directly visualises the conformation of tubulin that CKK recognises. The data quality readily allows discrimination of individual protofilaments and in many cases individual subunits (Fig. 5g), and provides critical 3D information about the MT ends.

Individual protofilament 3D trajectories within five minus and five plus ends (identified using previously established approaches ${ }^{29}$ (see Supplementary Fig.9a,b) were analyzed. Overall the individual MT ends are very heterogeneous, and there are no significant differences between minus and plus ends. However, their structures are not blunt but are also distinct from those of a MT end model constructed using available high resolution structures of curved tubulin (Supplementary Fig. 9c). First, plots of individual protofilament trajectories at both plus and minus ends show a range of curvatures and lengths of curved regions (see Fig. 5h for an example of a minus end). Second, while there is a wide variation of protofilament curvature, protofilaments at each end are on average less curved than in the model (Fig. 5i,j). The minus and plus end protofilaments are similar with respect to the length (18 $\pm 15 \mathrm{~nm}$ and $11 \pm 8 \mathrm{~nm}$ at the minus and plus ends, respectively) and extent of longitudinal curvature, which is consistent with previous cryo-EM studies in $2 \mathrm{D}^{30,31}$. This suggests that CKK minus-end preference does not depend on longitudinal curvature alone.

Intriguingly, adjacent protofilaments within a MT end retain connectivity with their neighbors (Fig. 5k, Supplementary Fig. 9d), even while the protofilaments spread away from the MT axis and the total protofilament number reduces. This behavior is in contrast to the MT end model in which protofilament separation is an essential consequence of their curvature and is more consistent with the classical "rams horns" of depolymerizing MTs ${ }^{31}$, to which CAMSAP does not bind (Fig. 5c). In other words, in non-depolymerizing MTs, protofilaments curving outwards from the MT axis flatten from a cylinder into gently curving sheet-like structures, which retain lateral connectivity $30,32,33$. Given that the CKK domain binds at the inter-protofilament interface, the tomographic data suggest that potential CKK binding sites are retained in the end structures we have characterised. To check whether CKK binding perturbs these curved sheet-like structures, we examined MT minus ends in the presence of CKK but detected no differences compared to the control MTs (Supplementary Fig. 9e-h). This analysis, when combined with our fluorescence-based localization of the CKK binding site, suggests the CKK prefers neither the extreme end nor the MT lattice but a transition region from the straight lattice to a curved and flattened polymer.

\section{The CKK domain inhibits MCAK-induced MT minus-end depolymerization by blocking its interaction with minus ends}

It has been shown that fly Patronin and three mammalian CAMSAPs can protect MT minus ends from the depolymerization by kinesin-136, 19, but the underlying mechanism is unknown. By superimposing HsMCAK motor structure onto our CKK-MT cryo-EM 
reconstruction, we noticed that CKK and HsMCAK would strongly clash with each other at a number of positions (Fig. 6a). This suggests that CKK and MCAK would compete for the binding sites at MT minus ends. To test this hypothesis, we measured in vitro disassembly of GMPCPP stabilized MTs by $50 \mathrm{nM}$ MCAK in the presence of different concentrations of CAMSAP1 $1_{\text {mini }}$ or the isolated CKK domain (Fig. 6b,c). CAMSAP1 $1_{\text {mini }}$ and CKK almost completely inhibited the minus-end binding and depolymerization of MCAK at $1.5 \mathrm{nM}$ and $38 \mathrm{nM}$, respectively, while the activity of MCAK on plus ends was largely unaffected at these concentrations (Fig. 6d,e). Above $1 \mu \mathrm{M}$ concentration, even though both CAMSAP $1_{\text {mini }}$ and CKK fully decorated MT shaft with lattice intensities higher than those observed at the minus ends at low concentrations, this decoration did not lead to a complete inhibition of MT plus-end accumulation and depolymerization by MCAK (Fig. 6d,e). This observation suggests that the CKK has the highest affinity for MT minus ends, followed by the MT lattice, and the lowest affinity for MT plus ends. The alternative mechanism - that displacement of MCAK from MT minus ends is due to CKK-mediated alteration of MT minus-end structure which would be less favorable for MCAK binding - is not supported by our cryo-EM images even at CKK concentration of $>50 \mu \mathrm{M}$, which was much higher than the ones used in our in vitro assays (Supplementary Fig. 9e-h). This result indicates that the competition between CKK and MCAK on MT minus ends is primarily based on steric hindrance.

\section{Discussion}

In this study, we have shown that the CKK is a highly conserved globular protein module that interacts with an inter-protofilament site between two tubulin dimers on the outer surface of MTs. Several lines of evidence suggest that an optimal site for CKK binding deviates from the inter-protofilament interface within a regular, straight MT structure. First, the decoration by CKK distorts the normal MT lattice generating a right-handed protofilament skew. Second, tubulin copolymerisation with CKK yields mainly non-MT, curved polymers. Third, the interaction between CKK and its binding site is intrinsically asymmetric, with the helical parts of CKK interacting more closely with the $\beta$-tubulin pairs, while its connectivity with the a-tubulin pair is less compact and could accommodate plasticity existing at minus ends. Fourth, the N1492A mutation increases affinity and subtly alters the CKK position relative to the MT, resulting in a deeper insertion between the protofilaments. The size and the chemical nature of the N1492 substituted side chain also affect CKK affinity for the MT lattice, suggesting that the optimal binding site for the wild type CKK domain might depend on specific but subtle conformational features of tubulin dimers at MT minus ends to allow a more buried fit between protofilaments. Fifth, highresolution microscopy showed that the high-affinity CKK binding region is located, on average, two tubulin dimers behind the outmost MT end. This binding site fits very well with the average position of the transition zone between straight protofilaments in the MT shaft and mildly curved tubulin sheets identified at MT ends by cryo-electron tomography.

At this transition zone, pairs of a-tubulins would be more laterally flattened and more flexible than the more constrained lattice-like $\beta$-tubulin pairs (Fig. 7a). Consistent with this, our structures show that the lattice-bound CKK already has tight shape complementarity and a large contact surface with the $\beta$-tubulin pairs, whilst the a-tubulin pairs surround the CKK 
more loosely. Conversely, at the plus end, $\beta$-tubulin pairs are expected to acquire a more flattened and flexible arrangement than a-tubulins. This would disfavor the interaction of CKK with the plus end, explaining its inability to effectively compete with MCAK at this location. In summary, flattening of $\beta$-tubulin pairs at the plus end could squeeze CKK out of the tubulin groove to avoid steric clashes, whilst flattening of a-tubulin pairs at the minus end could tighten their multiple contacts around the CKK. This leads to a binding preference for the minus end, some capacity to bind the straight MT lattice and low affinity for MT plus end. The lack of CKK binding at rapidly depolymerizing MT minus ends, where the lateral contacts between protofilaments are expected to be lost due to their strong curling, is consistent with the requirement for maintenance of lateral protofilament connectivity and/or more gentle longitudinal curvature for CAMSAP binding.

It is important to note that although our analysis showed that CKK is the only CAMSAP part which can autonomously recognise MT minus ends, some longer CAMSAP fragments, such as CAMSAP1 $1_{\text {mini }}$, had a higher affinity for the minus ends. These data indicate that CAMSAP regions outside of the CKK domain can contribute to the affinity and selectivity of the minus-end binding, and additional work will be needed to decipher the underlying mechanisms.

The MT minus-end binding mode of the CKK domain is distinct from those of other known MT tip-interacting proteins. The most conceptually simple mode of MT end recognition relies on the presence of unique epitopes of $a$ - and $\beta$-tubulin exposed on the outermost MT ends, as is the case for the $\gamma$-TuRC at the minus end ${ }^{5}$, and which likely applies to the centriole protein CPAP at MT plus ends ${ }^{34}$. However, the preference of most known MT regulators is based on recognition of certain MT lattice features that are different at MT ends compared to the MT shaft. For example, EB proteins bind at the corner of four tubulin dimers $^{35,36}$ and sense a GTP hydrolysis transition state of tubulin within the MT lattice at both plus- and minus ends ${ }^{37,38}$. Doublecortin (DCX), which also binds at the corner of four tubulin dimers ${ }^{39}$, can also act as a MT end-binding protein and is thought to be sensitive to the extent of protofilament longitudinal curvature ${ }^{40,41}$. MT depolymerizing kinesin-13s can also accumulate at MT ends, likely due to preference for the more curved or flexible conformations of tubulin located outside the lattice ${ }^{42,43}$. Importantly, none of these mechanisms is specific for the plus or the minus end.

CAMSAPs are similar to most other MT end-binding proteins in that they bind on the outer surface of MTs.: in contrast to $\gamma$-TuRC, which caps MTs, CAMSAP binding is compatible with further MT polymerization at the minus end ${ }^{18}$. CAMSAPs and $\gamma$-TuRCs thus do not directly compete for binding to MT minus ends; however, the restricted, lattice-like conformation of tubulin at $\gamma$-TuRC-capped minus ends likely prevents formation of CAMSAP-preferred binding regions. Importantly, in contrast to EBs, CAMSAP-MT association is insensitive to the hydrolysis state of the tubulin-bound nucleotide. Whereas the tubulin inter-dimer interface to which EBs bind changes conformation in response to nucleotide, the intra-dimer interface to which CKKs bind does not ${ }^{36,44}$. This accounts for the ability of CAMSAPs to recognize growing, GTP- as well as the GDP-bound minus ends generated by MT severing ${ }^{18}$. 
CAMSAP interaction with MT minus ends can be expected to have several consequences. First, by preferentially binding between laterally connected but curved protofilaments, the CKK - in the context of the full length protein - might inhibit formation of a regular lattice, explaining why CAMSAPs slow down MT minus-end polymerization ${ }^{18}$. Second, CKK binding at intra-dimer binding sites can also explain protection of MT minus ends from kinesin-13 driven depolymerization by direct steric inhibition (Fig. 7b).

Taken together, our data show that the unique MT-binding mode of the CKK domains enables CAMSAPs to combine the ability to recognize MT minus ends with the preservation of dynamic properties of these ends. This allows CAMSAPs to be rapidly recruited to MT minus ends generated by release from the site of MT nucleation, severing or breakage, protect them against disassembly by depolymerases and, by decorating polymerizing minus ends, form stretches of stabilized MT lattice. Future studies will show whether there are other proteins sharing the CKK's MT binding mode, or whether it is unique to this evolutionary ancient and widespread protein module.

\section{Online Methods}

\section{Sequence analysis}

For the detection of CKK homologs BLASTP/PSI-BLAST ${ }^{50}$ and phmmer/jackhmmer ${ }^{51}$ searches were performed online across the $\mathrm{nr}$ and UniProt databases, respectively, taking different sequences as query. Based on these hits, a multiple sequence alignment was made with MAFFT ${ }^{52}$, version v7.149b, option einsi make an HMM profile. This profile was used to search for other sequences containing the CKK domain across our own dedicated set of eukaryotic proteomes (Supplementary Table 1). The resulting full-length protein sequences can be found in Supplementary Sequence File.

\section{Protein expression and purification for crystallization}

The DNA encoding the Mus musculus CAMSAP3 CKK core domain (denoted CKK 3 core, residues 1121-1239; Uniprot: Q80VC9) was amplified from the Mus musculus CAMSAP3 CKK domain vector (denoted CKK3) (residues 1112-1252) and cloned into the pET-based bacterial expression vector PSTCm2 (with an N-terminal 6x His-tag) by the positive selection methods ${ }^{53}$. The CKK3 and the mutants for biophysics characterization were cloned into the pET28a vector. Protein production was performed in the Escherichia coli strain BL21(DE3) (Stratagene) in LB media containing $50 \mu \mathrm{g} / \mathrm{ml}$ kanamycin. When the one liter cultures had reached an $\mathrm{OD}_{600}$ of 0.6 at $37^{\circ} \mathrm{C}$, the media were cooled down to $20^{\circ} \mathrm{C}$ and the expression was induced with $1 \mathrm{mM}$ isopropyl 1 -thio- $\beta$-D-galactopyranoside (IPTG). The expression was conducted for 16 hours at $20^{\circ} \mathrm{C}$. After harvesting and washing the cells with Dulbecco PBS (Millipore), the cells were sonicated in the presence of the protease inhibitor cOmplete cocktail (Roche) in lysis buffer (50 mM HEPES, pH 8, supplemented with 500 $\mathrm{mM} \mathrm{NaCl}, 10 \mathrm{mM}$ imidazole, $2 \mathrm{mM} \beta$-mercaptoethanol, $0.1 \%$ bovine deoxyribonuclease I).

Proteins were purified by immobilized metal-affinity chromatography (IMAC) on a HisTrap HP Ni2+-Sepharose column (GE Healthcare) at $4{ }^{\circ} \mathrm{C}$ following the instructions of the manufacturer. The column was equilibrated in IMAC A buffer (50 mM HEPES, pH 8, 
supplemented with $500 \mathrm{mM} \mathrm{NaCl}, 10 \mathrm{mM}$ imidazole, $2 \mathrm{mM} \beta$-mercaptoethanol). Proteins were eluted by IMAC B buffer that contained $400 \mathrm{mM}$ imidazole after washing with $5 \%$ IMAC buffer B. In the case of $\mathrm{CKK} 3_{\text {core }}$, the $\mathrm{N}$-terminal His-tag was cleaved off by an inhouse produced HRV $3 \mathrm{C}$ protease in IMAC A buffer for 16 hours at $4{ }^{\circ} \mathrm{C}$. The cleaved sample was applied again on the IMAC column to separate cleaved from uncleaved protein.

Proteins were concentrated and loaded on a SEC HiLoad Superdex 75 16/60 column (GE Healthcare) that was equilibrated in $20 \mathrm{mM}$ Tris- $\mathrm{HCl}, \mathrm{pH} 7.5$, supplemented with $150 \mathrm{mM}$ $\mathrm{NaCl}$ and $1 \mathrm{mM}$ DTT. The fractions of the main peak were pooled and concentrated to 10 $\mathrm{mg} / \mathrm{ml}$. Protein quality and identity were assessed by SDS-PAGE and mass spectrometry, respectively.

\section{Protein expression and purification for cryo-EM}

Human CAMSAP1 wild type, N1492A CKK (residues 1474-1613) and mouse CAMSAP3 CKK (residues 1112-1252) were cloned into pET28a vector. Following the purification using Ni-NTA resin (Qiagen), proteins were further purified on an ion exchange column MonoS and gel filtration column Superose 6 (GE Healthcare). Purified proteins were concentrated to $\sim 20 \mathrm{mg} / \mathrm{ml}$ in BRB20 buffer.

\section{Protein expression and purification for in vitro assays}

All proteins used for Total Internal Reflection Fluorescence microscopy (TIRFM) (human CAMSAP1 CKK (residues 1474-1613) and CAMSAP $1_{\text {mini }}$ (residues 1227-1613), fly CKK (residues 1547-1689), worm CKK (residues 982-1128), Tetrahymena thermophila CKK (residues 1635-1770), Trichomonas vaginalis CKK (residues 650-795), Naegleria gruberi CKK (residues 612-788), Phytophthora infestans CKK (residues 1120-1168), human MCAK full length and human KIF5B (residues 1-560)) were expressed in HEK293T cells using modified pTT5 expression vector (Addgene, $\# 44006,{ }^{54}$ ) bearing strep-GFP or strepSNAP tag at either $\mathrm{N}$ - or $\mathrm{C}$ terminus of the protein. The CKK cDNA sequences encoding $T$. thermophila, $T$. vaginalis, $N$. gruberi were codon optimized for human cell expression and synthesized as gBlock from IDT. The CKK of $P$. infestans was obtained by PCR from genomic DNA (a gift from Dr. F. Govers, Wageningen University). Fly and worm Patronin cDNAs were kindly provided by Dr. V. Gelfand (Northwestern University) and Dr. M. Harterink (Utrecht University), respectively. In CAMSAP1mini-GFP protein, the GFP tag was inserted directly after the C-terminus of the CKK domain followed by a short flexible linker (CAMSAP1 residues 1227-1613-GGSGGS-GFP)

Cells from one $15 \mathrm{~cm}$ dish collected after $36 \mathrm{hrs}$ transfection were lysed in $900 \mu \mathrm{llysis}$ buffer (50 mM HEPES, $300 \mathrm{mM} \mathrm{NaCl}, 0.5 \%$ Triton X-100, pH 7.4) supplemented with protease inhibitors (Roche). After clearing debris by centrifugation, cell lysates were incubated with $100 \mu \mathrm{l}$ StrepTactin beads (GE Healthcare) for 45 mins. Beads were washed 5 times with lysis buffer without protease inhibitors and twice with the wash buffer $(50 \mathrm{mM}$ HEPES, $150 \mathrm{mM} \mathrm{NaCl}$ and $0.01 \%$ Triton X-100). The proteins were eluted in $60 \mu$ elution buffer (50 mM HEPES, $150 \mathrm{mM} \mathrm{NaCl}, 0.01 \%$ Triton X-100 and $2.5 \mathrm{mM}$ desthiobiotin).

To label SNAP tagged proteins with Alexa-647 dye (NEB), 20-40 $\mu \mathrm{M}$ dye was incubated with proteins on beads for $1 \mathrm{hr}$ between the washing and elution steps. After extensive 
washing steps, proteins were eluted in elution buffer containing $300 \mathrm{mM}$ instead of $150 \mathrm{mM}$ $\mathrm{NaCl}$.

HEK293T cell line was obtained from ATCC, was not found in the database of commonly misidentified cell lines maintained by ICLAC and NCBI BioSample, was not authenticated and was negative for mycoplasma contamination.

\section{Total Internal Reflection Fluorescence microscopy (TIRFM)}

TIRFM was performed on an inverted research microscope Nikon Eclipse Ti-E (Nikon) with the perfect focus system (PFS) (Nikon), equipped with the Nikon CFI Apo TIRF 100× 1.49 N.A. oil objective (Nikon), Photometrics Evolve 512 EMCCD (Roper Scientific) and controlled with the MetaMorph 7.7 software (Molecular Devices). Images were projected onto the chip of Evolve 512 camera with intermediate lens 2.5X (Nikon C mount adapter 2.5X). To keep in vitro samples at $30^{\circ} \mathrm{C}$, we used stage top incubator INUBG2E-ZILCS (Tokai Hit).

For excitation we used $491 \mathrm{~nm} 100 \mathrm{~mW}$ Stradus (Vortran), 561nm 100mW Jive (Cobolt) and $642 \mathrm{~nm} 110 \mathrm{~mW}$ Stradus (Vortran). We used ET-GFP 49002 filter set (Chroma) for imaging of proteins tagged with GFP, ET-mCherry 49008 filter set (Chroma) for imaging XRhodamine labelled tubulin or mCherry-EB3 and ET-405/488/561/647 for imaging SNAPAlexa647. For simultaneous imaging of green and red fluorescence, we used the triple-band TIRF polychroic ZT405/488/561rpc (Chroma) and the triple-band laser emission filter ZET405/488/561m (Chroma), mounted in the metal cube (Chroma, 91032) together with Optosplit III beamsplitter (Cairn Research Ltd, UK) equipped with a double emission filter cube configured with ET525/50m, ET630/75m and T585LPXR (Chroma). We used sequential acquisition for triple colour imaging experiments.

\section{In vitro MT assays}

The in vitro assays with dynamic MTs were performed under the same conditions as described previously ${ }^{18}$. Briefly, after functionalizing coverslips by sequentially incubating them with $0.2 \mathrm{mg} / \mathrm{ml}$ PLL-PEG-biotin (Susos AG, Switzerland) and $1 \mathrm{mg} / \mathrm{ml}$ neutravidin (Invitrogen) in MRB80 buffer, GMPCPP-stabilized MT seeds were attached to coverslips through biotin-neutravidin interactions. Flow chambers were further blocked with $1 \mathrm{mg} / \mathrm{ml}$ K-casein. The reaction mix with purified proteins (MRB80 buffer supplemented with $20 \mu \mathrm{M}$ porcine brain tubulin, $0.5 \mu \mathrm{M} \mathrm{X}$-rhodamine-tubulin, $75 \mathrm{mM} \mathrm{KCl}, 1 \mathrm{mM} \mathrm{GTP}, 0.2 \mathrm{mg} / \mathrm{ml} \kappa$ casein, $0.1 \%$ methylcellulose and oxygen scavenger mix (50 mM glucose, $400 \mu \mathrm{g} / \mathrm{ml}$ glucose oxidase, $200 \mu \mathrm{g} / \mathrm{ml}$ catalase and $4 \mathrm{mM}$ DTT) was added to the flow chamber after centrifugation. The flow chamber was sealed with vacuum grease, and dynamic MTs were imaged immediately at $30^{\circ} \mathrm{C}$ using a TIRF microscope.

The conditions for MT depolymerisation assay were essential the same as in the assays with dynamic MTs except that tubulin proteins were not included and the reaction mix was optimized to image MT depolymerisation (MRB80 buffer supplemented with $100 \mathrm{mM} \mathrm{KCl}$, $1 \mathrm{mM}$ GTP, $1 \mathrm{mM}$ ATP, $0.2 \mathrm{mg} / \mathrm{ml} \kappa$-casein and oxygen scavenger mix). All tubulin products were from Cytoskeleton. 


\section{Quantification of the intensity of the wild type and mutant CAMSAP1 mini $_{\text {in }}$ on dynamic MTs}

To quantify the minus-end and lattice intensity of CAMSAP $1_{\text {mini }}$ in a time-lapse movie, kymographs were generated in ImageJ using KymoResliceWide plug-in. The minus-end positions were marked by 5-pixel wide linear ROIs corresponding to CAMSAP 1 mini signals. The maximum intensity within 5-pixel region along the spatial axis of the kymograph was measured by using a Macro written in ImageJ.

\section{Determination of CAMSAP1 position on MT ends}

To determine the position of CAMSAP $1_{\text {mini }}$ relative to the MT minus end, we

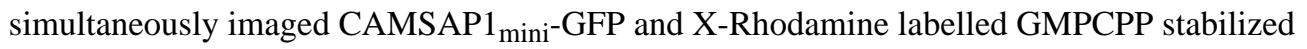
MTs using a beam splitter. Spatial registration between two channels was performed by Bspline point based transform (MathWorks File Exchange: 20057-b-spline-grid--image-andpoint-based-registration) using images of a calibration grid featuring $500 \mathrm{~nm}$ diameter transparent circles separated by $2 \mu \mathrm{m}$ distance (Compugraphics, UK). In order to achieve better spatial sampling of imaging area, an image of "denser" grid was obtained by moving the stage eight times with the step of $0.25 \mu \mathrm{m}$ in both $\mathrm{x}$ and $\mathrm{y}$ direction during calibraion. After channel's registration, we extracted one-dimensional intensity profiles along MT axis for both CAMSAP $1_{\operatorname{mini}}$ and MT channels. The profiles were fitted to the models previously used for XMAP215 and MT ends ${ }^{28}$. Briefly, MT end axis profile was modelled as an error function, which is the result of convolution between step function representing density of tubulin molecules distribution in a MT and a Gaussian representing the optical point spread function (PSF) of the microscope. CAMSAP1 $1_{\text {mini }}$ axis profile was modelled as the combination of Gaussian peak (representing convoluted point like accumulation at the edge of the minus-end) and error function (accounting for its weak lattice binding) with different weights. The PSF size estimation (GFP channel: $108 \mathrm{~nm}$; x-Rhodamine channel: $125 \mathrm{~nm}$ ) was obtained from two colour images of $100 \mathrm{~nm}$ diameter fluorescence beads (TetraSpeck, Invitrogen).

The overall displacement error between point images in two channels after registration was estimated to be $\sim 2 \mathrm{~nm}$. It based on acquiring an extra set of calibration grid images and calculating the average distance between corresponding spots in two channels after registration. The average error of fitting for the position parameters is about $5 \mathrm{~nm}$ and $6 \mathrm{~nm}$ for CAMSAP1 (peak position) and MT (edge position), respectively. Thus, according to the propagation of uncertainty the estimated error of a distance between the peak of CAMSAP1 distribution and the edge of MT minus-end is about $\sqrt{2^{2}+5^{2}+6^{2}} \approx 8 \mathrm{~nm}$.

\section{Structure determination of the CAMSAP3 CKK domain}

$\mathrm{CKK}_{3}$ core was concentrated to $14 \mathrm{mg} / \mathrm{ml}$ and TCEP to a final concentration of $5 \mathrm{mM}$ was added. The screening of crystallization conditions was performed using a Mosquito robot (TTP Labtech) in 96 wells plate using the vapor diffusion hanging drop method. In drops of a 1:1 (200 nl each) mixture of $\mathrm{CKK} 3_{\text {core }}$ and mother liquor (100 mM citric acid, $\mathrm{pH} \mathrm{5,1} \mathrm{M}$ $\mathrm{NaCl}$ ) crystals with a diameter of $100 \mu \mathrm{m}$ appeared after one week. The crystals were cryoprotected by transfer into mother liquor supplemented with $10 \%$ glycerol and were flash frozen in liquid nitrogen. 
Diffraction data were collected at the X06DA macromolecular crystallography beamline at the Swiss Light Source (Paul Scherrer Institut) at a wavelength of $1 \AA$. The data were indexed with LABELIT ${ }^{55}$, refined and integrated in XDS ${ }^{56}$. The crystals diffracted up to 1.4 $\AA$ at a wavelength and the crystals belonged to the space group I422 with a $=96.4 \AA, \mathrm{b}=$ $96.4 \AA$ and $\mathrm{c}=63.3 \AA$. The structure was solved by molecular replacement using PHASER $^{57}$ with the available NMR structure (PDB ID 1UGJ) as a search model. Several rounds of manual model building in COOT $^{58}$ and refinement in PHENIX.refine ${ }^{59}$ and REFMAC5 ${ }^{60}$ produced a final model with satisfactory R-work/R-free. The structure was validated by MolProbity and the wwPDB Validation Service. Figures were created using PyMOL ${ }^{61}$. Data collection and refinement statistics are given in Table 1.

\section{Circular dichroism (CD) spectroscopy}

CD spectra of CKK3 and derived mutants were recorded on a Chirascan-Plus CD instrument (Applied Photophysics Ltd.) equipped with a computer-controlled Peltier element using a cuvette of $1 \mathrm{~mm}$ optical path length. $0.25 \mathrm{mg} / \mathrm{ml}$ of protein sample was applied in PBS buffer. Thermal unfolding profiles were measured at $206 \mathrm{~nm}$ by continuous heating at $1{ }^{\circ} \mathrm{C}$ $\min -1$.

\section{MT pelleting assays}

MT pelleting assays of purified proteins were performed as previously described ${ }^{62}$. Briefly, $10 \mathrm{mg} / \mathrm{ml}$ bovine brain tubulin was diluted in 1x BRB35 buffer (35 mM K-PIPES, pH 6.8, supplemented with $1 \mathrm{mM}$ EGTA, $1 \mathrm{mM} \mathrm{MgCl} 2,1 \mathrm{mM}$ DTT) to $1 \mathrm{mg} / \mathrm{ml}$. After the addition of $0.5 \mathrm{mM}$ GTP, the sample was incubated on ice for 5 minutes. MT polymerization was started by the transfer to $37^{\circ} \mathrm{C}$. After 10 minutes, $0.1 \mu \mathrm{M}, 1 \mu \mathrm{M}$ and $10 \mu \mathrm{M}$ paclitaxel were added stepwise with incubation times of 5 minutes each. Pelleting assays in the presence of CAMSAP3-CKK were performed by mixing $3.8 \mu \mathrm{M}$ of taxol-stabilized MTs with an equimolar ratio of the CAMSAP3-CKK in the presence of different amounts of sodium chloride concentrations. As controls, taxol-stabilized MTs or the CAMSAP3-CKK was applied alone. Samples were applied onto a taxol-glycerol cushion that contained 55\% 2x BRB35, 44\% Glycerol and 6\% $2 \mathrm{mM}$ paclitaxel. After centrifugation at $174,500 \mathrm{x} \mathrm{g}$ for 30 min at $25{ }^{\circ} \mathrm{C}$, an aliquot was taken from the supernatant. After removing the supernatant the pellet was resuspended into SDS sample buffer. Samples were loaded and analyzed on Coomassie stained $15 \%$ SDS gel.

\section{Cryo-EM methods summary (details are provided in the Cryo-EM Methods Supplemenatary note)}

Single particle cryo-EM data of MT-CAMSAP3 CKK domain complexes were collected on a Tecnai G2 Polara (FEI), while data of MT-CAMSAP1 CKK domain complexes were collected on a Tecnai F20 (FEI), both using a DE20 direct electron detector (Direct Electron). Data of MT-CAMSAP1 N1492A mutant CKK domain complexes were collected on a Tecnai G2 Polara using a K2 direct electron detector (Gatan) operating in counting mode. Cryo-electron tomography single-axis tilt series of MT ends were collected on a Tecnai G2 Polara on a K2 summit direct electron detector (Gatan) operating in counting mode. MTs for single particle reconstruction were boxed manually, and were input to a set of custom-designed semi-automated single-particle processing scripts utilizing Spider and 
Frealign as described previously ${ }^{63,64}$. The final dataset sizes and resolutions are reported in Table 2. For the cryo-ET data, tilt series were processed and tomograms generated using IMOD's Etomo graphical user interface (v4.7.15).

\section{CKK domain-MT pseudo-atomic model building}

The X-ray structure of the CAMSAP3-CKK domain (PDB ID 5LZN) was rigidly fitted into CKK domain density of CAMSAP3-CKK domain map using UCSF Chimera's 'fit in map' tool. A homology model of CAMSAP1-CKK (residues 1474-1600) was generated using MODELLER $^{65}$, based on the CAMSAP3-CKK crystal structure as template. In order to model missing loops and terminal regions for both CKKs, the deposited NMR structure of the CAMSAP3 CKK domain (PDB ID 1UGJ) was used as template. CKK models were selected based on MODELLER's statistical potentials score - zDOPE $^{66}$. The model of CAMSAP1-CKK was rigidly fitted into its density map and for both CKKs, the local fits of secondary-structural elements and loops were scored with a local correlation score using TEMPy (SCCC, ${ }^{67}$. Loop regions which had low SCCC scores, were further optimized. For each of these loops, 200 loop-models were generated using MODELLER loop optimization protocol $^{68}$ and a top-scoring conformation (based on SCCC) was selected. To create the N1492A CAMSAP1 CKK model, the point-mutation on the wild type CAMSAP1 model was performed in $\mathrm{Coot}^{69}$ and this was rigidly fitted into the corresponding mutant CKK density.

Final CKK fits were combined with rigid fits (using Chimera's 'fit in map') of two tubulin dimers from the structure of the paclitaxel stabilized-MT ${ }^{44}$ (PDB ID 3J6G). The rigid fitted models were already a good fit to experimental density, therefore the models underwent final refinement to their Bfactor sharpening resolutions (Table 2) with NCS restraints using the phenix.real_space_refine tool in Phenix ${ }^{70}$ to resolve clashes and improve model geometry. The MolProbity validation indicated zero Ramachandran outliers, 98.1\% Ramachandran favored residues, zero rotamer outliers and a clash score of 0.56 .

\section{Molecular dynamics simulations}

4000 cycles of energy minimization without any positional restrains were performed on the refined structural complexes. Two consecutive molecular dynamics simulation runs of 10ps and 200ps were then employed to increase the temperature from $100 \mathrm{~K}$ to $300 \mathrm{~K}$ and to equilibrate the systems at $300 \mathrm{~K}$. Four replicate production runs of $40 \mathrm{~ns}$ each were then performed starting from the equilibrated system configurations. The simulations were run at constant temperature $(300 \mathrm{~K})$ and constant pressure (1atm) using a $2 \mathrm{fs}$ time step. Periodic boundary conditions and full particle-mesh Ewald electrostatic were used. A $12 \AA$ cutoff value was applied to truncate the non-bonded interactions. The SHAKE algorithm was used to constrain the covalent bonds formed by hydrogen atoms. Each replicate simulation was run with different random starting velocities.

\section{MM/GBSA calculations}

Molecular mechanics with generalized Born and surface area solvation (MM/GBSA) calculations were performed with the GBOBC model (6) in AMBER 12 (1). For each molecular dynamics simulation, pairwise energetic interactions values (flag idecomp=4) 
were scaled by the average number of CKK domain - tubulin dimers contacts (51 contacts) and averaged over four replica simulations.

\section{NMR sample preparation and experiments}

Uniformly $\left[{ }^{13} \mathrm{C},{ }^{15} \mathrm{~N}\right]$ variants of CKK were produced in Escherichia coli strain Rosetta 2 in M9 minimum media containing $25 \mu \mathrm{g} / \mathrm{ml}$ kanamycin and $35 \mu \mathrm{g} / \mathrm{ml}$ chloramphenicol. The cells were induced with $0.3 \mathrm{mM}$ IPTG at $25{ }^{\circ} \mathrm{C}$ for overnight after $\mathrm{OD}_{600}$ reached 0.6. Proteins were purified as described above with phosphate buffer instead of HEPES buffer. After purification, proteins were loaded onto a SEC HiLoad Superdex 75 26/60 column (GE Healthcare), which was equilibrated in $40 \mathrm{mM}$ phosphate buffer with $150 \mathrm{mM} \mathrm{NaCl}$ and 1 $\mathrm{mM}$ DTT, $\mathrm{pH}$ 7.0. Proteins were then concentrated and used for solution-state NMR measurement supplemented with $5 \% \mathrm{D}_{2} \mathrm{O}$, or ssNMR sample preparation.

For ssNMR experiments, $\left[{ }^{13} \mathrm{C},{ }^{15} \mathrm{~N}\right]$-CAMSAP3-CKK/MT complex was prepared. $20 \mathrm{mg}$ of lyophilized tubulin was first dissolved in BRB80 buffer to make a final concentration of 2 $\mathrm{mg} / \mathrm{ml}$. Tubulin was then polymerized with addition of $20 \mu \mathrm{M}$ paclitaxel for 30 minutes at $37^{\circ} \mathrm{C}$. Paclitaxel-stabilized MTs were centrifuged at 55,000 rpm (Beckman TLA-55 rotor) at $30{ }^{\circ} \mathrm{C}$ for 30 minutes. The pellet was resuspended in warm BRB80 buffer and labeled CKK domain was added to the final concentration of $65.3 \mu \mathrm{M}$ (CKK:tubulin ratio 4:1). The mixture was incubated at $37^{\circ} \mathrm{C}$ for 30 minutes and then centrifuged at 55,000 rpm (Beckman TLA-55 rotor) at $30{ }^{\circ} \mathrm{C}$ for 30 minutes. The pellet was washed with phosphate buffer without disturbing the pellet. Finally, the pellet was transferred and packed into a 3.2 $\mathrm{mm}$ rotor.

Resonance assignments were obtained from previous results (PDB ID 1UGJ) and additional solution-state NMR experiments on free CKK were recorded on a $600 \mathrm{MHz}$ spectrometer (Bruker Biospin) to assign missing residues (2D HSQCs, 3D HNCA, HNCO, HNCACB, $\mathrm{CBCA}(\mathrm{CO}) \mathrm{NH}, \mathrm{HAHB}(\mathrm{CO}) \mathrm{NH}, \mathrm{hCCH}-\mathrm{DIPSI})$. Solid-state NMR experiments involved two-dimensional NCA and CC Proton-driven spin diffusion (PDSD) experiments (temperature $260 \mathrm{~K}$, MAS rate $14 \mathrm{kHz}$ ) as well as additional 2Q-1Q experiments (temperature $268 \mathrm{~K}$, MAS $10 \mathrm{kHz}$ ). Mixing schemes employed SPECIFIC-CP transfers $^{71}$ as well as SPC $5^{72}$ and Spin diffusion under weak coupling conditions ${ }^{73}$ for longer PDSD mixing times. Data were recorded on a $950 \mathrm{MHz}$ standard-bore spectrometer (Bruker Biospin) equipped with a $3.2 \mathrm{~mm}$ triple-channel MAS HCN probe. ssNMR data were analyzed using NMR assignments obtained on free CKK as a reference. Resolved residues for which signal matched with ssNMR data within $0.5 \mathrm{ppm}$ in $13 \mathrm{C}$ and $1 \mathrm{ppm}$ in $15 \mathrm{~N}$ dimensions ${ }^{74}$ were considered as unperturbed, while resolved residues for which signal matched with ssNMR data within $1 \mathrm{ppm}$ in the indirect dimension and $0.5 \mathrm{ppm}$ in the direct dimension in the CC PDSD experiments were considered as unperturbed. Larger deviations were considered as altered and indicated in red.

\section{Analysis of CKK domain binding to subtilisin-treated MTs}

Paclitaxel-stabilized MTs were prepared containing 10\% rhodamine tubulin, 10\% biotinylated tubulin, and $80 \%$ unlabelled tubulin (Cytoskeleton, Inc.), then treated \pm 0.1 $\mathrm{mg} / \mathrm{ml}$ subtilisin for $30 \mathrm{~min}$ to remove predominantly $\beta$-tubulin C-terminal tails (CTTs), 
checked by western blot. The reaction was stopped by the addition of $10 \mathrm{mM}$ pefabloc, and MTs were isolated by centrifugation. GFP-CKK domain binding was analyzed by TIRFM, using flow chambers assembled from plasma-cleaned glass coverslips and microscope slides. Chambers were incubated sequentially with $1 \mathrm{mg} / \mathrm{ml}$ PLL-PEG biotin (Susos AG), block solution (1\% plurionic F-127, $4 \mathrm{mg} / \mathrm{ml}$ casein), $0.5 \mathrm{mg} / \mathrm{ml}$ neutravidin, and MTs \pm CTTs (as indicated). Each incubation was followed by two washes with MRB80 buffer (80 mM PIPES, $4 \mathrm{mM} \mathrm{MgCl}$, and $1 \mathrm{mM}$ EGTA [pH 6.8]) supplemented with $80 \mathrm{mM} \mathrm{KCl}, 20$ $\mu \mathrm{M}$ paclitaxel, $4 \mathrm{mM}$ DTT and $2 \mathrm{mg} / \mathrm{ml}$ casein. The final binding reaction contained $200 \mathrm{nM}$ CKK-GFP in MRB80 with $80 \mathrm{mM} \mathrm{KCl}, 20 \mu \mathrm{M}$ taxol, $4 \mathrm{mM}$ DTT and $2 \mathrm{mg} / \mathrm{ml}$ casein and an oxygen scavenger mix (400 $\mu \mathrm{g} / \mathrm{ml}$ glucose oxidase, $200 \mu \mathrm{g} / \mathrm{ml}$ catalase). TIRFM was performed on an Eclipse Ti-E inverted microscope with a Perfect Focus System, CFI Apo TIRF 1.49 N.A. oil objective, H-TIRF module and LU-N4 laser unit (Nikon). Images were recorded with a $100 \mathrm{~ms}$ exposure on an iXon DU888 Ultra EMCCD camera (Andor) controlled with NIS-Elements AR Software (Nikon).

\section{Statistics and Reproducibility}

The precise $\mathrm{p}$ values for Mann-Whitney U test were calculated in Matlab (MathWorks). All data shown are mean \pm SD. The sample size is indicated in the figure legends. All data presented in this study were either averages or representative data from at least two independent experiments. For the ssNMR experiments, all spectra were recorded twice on one sample and they were consistent. The sample did not alter by measuring the 1D H-C CP over time. A Life Sciences Reporting Summary for this article is available online.

\section{Code Availability}

The computer codes used in this study are available from the corresponding authors on request.

\section{Data Availability}

The structure of CKK3 $3_{\text {core }}$ was deposited in the PDBe databank under accession code 5LZN. The CKK-MT models along with their corresponding electron density maps are deposited in the PDB (CAMSAP1-CKK-MT, PDB: 5M54, CAMSAP1-N1492A-CKK-MT, PDB: 5M5C and CAMSAP3-CKK-MT, PDB: 5M50) and EMDB respectively (CAMSAP1-CKK-MT, EMDB: EMD-4156, CAMSAP1-N1492A-CKK-MT, EMDB: EMD-3444 and CAMSAP3CKK-MT, EMDB: EMD-4154). NMR data were deposited in the Biological Magnetic Resonance Bank, BMRB ID 27234.

All data that support the conclusions are available from the authors on request, and/or available in the manuscript itself. Source data for the figures 1, 3, 4, 5 and 6 and supplementary figures S1, S4, S7, S8 and S9 can be found in Supplementary Table S2.

\section{Supplementary Material}

Refer to Web version on PubMed Central for supplementary material. 


\section{Acknowledgements}

We thank F. Govers (Wageningen University, the Netherlands), V. Gelfand (Northwestern University, USA) and M. Harterink (Utrecht University, the Netherlands) for sharing reagents, E. Katrukha for advice on the processing of fluorescence microscopy data, R. Boelens for providing access to the solution-state NMR instrumentation and S. Yokoyama and A. Nomura for sharing NMR resonance assignments of free CKK. A.A. is supported by an ERC Synergy grant 609822. J.A., C.A.M. (MR/J000973/1), A-P.J. and M.T. (MR/M019292/1) are supported by the Medical Research Council, UK. A.J.R. is supported by a Sir Henry Dale Fellowship from the Wellcome Trust and Royal Society (104196/Z/14/Z). G.S. and B.J.G. are supported by the National Institutes of Health (R01GM070862) and Startup Funds from the University of Michigan, Ann Arbor. M.B., Y.L. (grant 718.015.001) and K.H. (grant 184.032.207) where supported by the Netherlands Organization for Scientific Research (NWO). Solid-state NMR Experiments were supported by uNMR-NL, an NWO-funded National Roadmap Large-scale Facility of the Netherlands (grant 184.032.207). M.O.S. is supported by grants from the Swiss National Science Foundation (31003A_166608) and from SystemsX.ch (RTD-TubeX).

\section{References}

1. Howard J \& Hyman AA Dynamics and mechanics of the microtubule plus end. Nature 422, $753-$ 758. (2003). [PubMed: 12700769]

2. Akhmanova A \& Steinmetz MO Control of microtubule organization and dynamics: two ends in the limelight. Nat Rev Mol Cell Biol 16, 711-726 (2015). [PubMed: 26562752]

3. Dammermann A, Desai A \& Oegema K The minus end in sight. Curr Biol 13, R614-624 (2003). [PubMed: 12906817]

4. Akhmanova A \& Hoogenraad CC Microtubule minus-end-targeting proteins. Curr Biol 25, R162171 (2015). [PubMed: 25689915]

5. Kollman JM, Merdes A, Mourey L \& Agard DA Microtubule nucleation by gamma-tubulin complexes. Nat Rev Mol Cell Biol 12, 709-721 (2011). [PubMed: 21993292]

6. Goodwin SS \& Vale RD Patronin regulates the microtubule network by protecting microtubule minus ends. Cell 143, 263-274 (2010). [PubMed: 20946984]

7. Derivery E et al. Polarized endosome dynamics by spindle asymmetry during asymmetric cell division. Nature 528, 280-285 (2015). [PubMed: 26659188]

8. Wang H, Brust-Mascher I, Civelekoglu-Scholey G \& Scholey JM Patronin mediates a switch from kinesin-13-dependent poleward flux to anaphase B spindle elongation. J Cell Biol 203, 35-46 (2013). [PubMed: 24100293]

9. Khanal I, Elbediwy A, Diaz de la Loza MD, Fletcher GC \& Thompson BJ. Shot and Patronin polarise microtubules to direct membrane traffic and biogenesis of microvilli in epithelia. J Cell Sci 129, 2651-2659 (2016). [PubMed: 27231092]

10. Toya $\mathrm{M}$ et al. CAMSAP3 orients the apical-to-basal polarity of microtubule arrays in epithelial cells. Proc Natl Acad Sci U S A 113, 332-337 (2016). [PubMed: 26715742]

11. Wang $\mathrm{S}$ et al. NOCA-1 functions with gamma-tubulin and in parallel to Patronin to assemble noncentrosomal microtubule arrays in C. elegans. Elife 4, e08649 (2015). [PubMed: 26371552]

12. Zheng $\mathrm{J}$ et al. Marshalin, a microtubule minus-end binding protein, regulates cytoskeletal structure in the organ of Corti. Biol Open 2, 1192-1202 (2013). [PubMed: 24244856]

13. Nashchekin D, Fernandes AR \& St Johnston D Patronin/Shot Cortical Foci Assemble the Noncentrosomal Microtubule Array that Specifies the Drosophila Anterior-Posterior Axis. Dev Cell 38, 61-72 (2016). [PubMed: 27404359]

14. Yau KW et al. Microtubule minus-end binding protein CAMSAP2 controls axon specification and dendrite development. Neuron 82, 1058-1073 (2014). [PubMed: 24908486]

15. Richardson CE et al. PTRN-1, a microtubule minus end-binding CAMSAP homolog, promotes microtubule function in Caenorhabditis elegans neurons. Elife 3, e01498 (2014). [PubMed: 24569477]

16. Marcette JD, Chen JJ \& Nonet ML The Caenorhabditis elegans microtubule minus-end binding homolog PTRN-1 stabilizes synapses and neurites. Elife 3, e01637 (2014). [PubMed: 24569480]

17. Chuang $M$ et al. The Microtubule Minus-End-Binding Protein Patronin/PTRN-1 Is Required for Axon Regeneration in C. elegans. Cell Rep 9, 874-883 (2014). [PubMed: 25437544] 
18. Jiang K et al. Microtubule minus-end stabilization by polymerization-driven CAMSAP deposition. Dev Cell 28, 295-309 (2014). [PubMed: 24486153]

19. Hendershott MC \& Vale RD Regulation of microtubule minus-end dynamics by CAMSAPs and Patronin. Proc Natl Acad Sci U S A 111, 5860-5865 (2014). [PubMed: 24706919]

20. Tanaka N, Meng W, Nagae S \& Takeichi M Nezha/CAMSAP3 and CAMSAP2 cooperate in epithelial-specific organization of noncentrosomal microtubules. Proc Natl Acad Sci U S A 109, 20029-20034 (2012). [PubMed: 23169647]

21. Baines AJ et al. The CKK domain (DUF1781) binds microtubules and defines the CAMSAP/ssp4 family of animal proteins. Mol Biol Evol 26, 2005-2014 (2009). [PubMed: 19508979]

22. Williams TA Evolution: rooting the eukaryotic tree of life. Curr Biol 24, R151-152 (2014). [PubMed: 24556435]

23. Chen $\mathrm{S}$ et al. High-resolution noise substitution to measure overfitting and validate resolution in $3 \mathrm{D}$ structure determination by single particle electron cryomicroscopy. Ultramicroscopy 135,24 35 (2013). [PubMed: 23872039]

24. Kumar A et al. Interaction of epothilone B (patupilone) with microtubules as detected by twodimensional solid-state NMR spectroscopy. Angew Chem Int Ed Engl 49, 7504-7507 (2010). [PubMed: 20809556]

25. Yan S et al. Atomic-resolution structure of the CAP-Gly domain of dynactin on polymeric microtubules determined by magic angle spinning NMR spectroscopy. Proc Natl Acad Sci U S A 112, 14611-14616 (2015). [PubMed: 26604305]

26. Scarabelli G et al. Mapping the Processivity Determinants of the Kinesin-3 Motor Domain. Biophys J 109, 1537-1540 (2015). [PubMed: 26488644]

27. Gardner MK et al. Model Convolution: A Computational Approach to Digital Image Interpretation. Cell Mol Bioeng 3, 163-170 (2010). [PubMed: 20461132]

28. Maurer SP et al. EB1 accelerates two conformational transitions important for microtubule maturation and dynamics. Curr Biol 24, 372-384 (2014). [PubMed: 24508171]

29. Chretien D, Kenney JM, Fuller SD \& Wade RH Determination of microtubule polarity by cryoelectron microscopy. Structure 4, 1031-1040 (1996). [PubMed: 8805589]

30. Chretien D, Fuller SD \& Karsenti E Structure of growing microtubule ends: two-dimensional sheets close into tubes at variable rates. J Cell Biol 129, 1311-1328 (1995). [PubMed: 7775577]

31. Mandelkow EM, Mandelkow E \& Milligan RA Microtubule dynamics and microtubule caps: a time-resolved cryo-electron microscopy study. J Cell Biol 114, 977-991 (1991). [PubMed: 1874792]

32. Guesdon A et al. EB1 interacts with outwardly curved and straight regions of the microtubule lattice. Nat Cell Biol 18, 1102-1108 (2016). [PubMed: 27617931]

33. Janosi IM, Chretien D \& Flyvbjerg H Modeling elastic properties of microtubule tips and walls. Eur Biophys J 27, 501-513 (1998). [PubMed: 9760731]

34. Sharma A et al. Centriolar CPAP/SAS-4 Imparts Slow Processive Microtubule Growth. Dev Cell 37, 362-376 (2016). [PubMed: 27219064]

35. Maurer SP, Fourniol FJ, Bohner G, Moores CA \& Surrey T EBs recognize a nucleotide-dependent structural cap at growing microtubule ends. Cell 149, 371-382 (2012). [PubMed: 22500803]

36. Zhang R, Alushin GM, Brown A \& Nogales E Mechanistic Origin of Microtubule Dynamic Instability and Its Modulation by EB Proteins. Cell 162, 849-859 (2015). [PubMed: 26234155]

37. Maurer SP, Bieling P, Cope J, Hoenger A \& Surrey T GTPgammaS microtubules mimic the growing microtubule end structure recognised by end-binding proteins (EBs). Proc Natl Acad Sci U S A 109, 3988-3993 (2011).

38. Zanic M, Stear JH, Hyman AA \& Howard J EB1 recognizes the nucleotide state of tubulin in the microtubule lattice. PLoS One 4, e7585 (2009). [PubMed: 19851462]

39. Fourniol FJ et al. Template-free 13-protofilament microtubule-MAP assembly visualized at $8 \mathrm{~A}$ resolution. J Cell Biol 191, 463-470 (2010). [PubMed: 20974813]

40. Bechstedt S, Lu K \& Brouhard GJ Doublecortin Recognizes the Longitudinal Curvature of the Microtubule End and Lattice. Curr Biol (2014). 
41. Ettinger A, van Haren J, Ribeiro SA \& Wittmann T Doublecortin Is Excluded from Growing Microtubule Ends and Recognizes the GDP-Microtubule Lattice. Curr Biol 26, 1549-1555 (2016). [PubMed: 27238282]

42. Desai A, Verma S, Mitchison TJ \& Walczak CE Kin I kinesins are microtubule-destabilizing enzymes. Cell 96, 69-78 (1999). [PubMed: 9989498]

43. Moores CA et al. A mechanism for microtubule depolymerization by KinI kinesins. Mol Cell 9, 903-909 (2002). [PubMed: 11983180]

44. Alushin GM et al. High-resolution microtubule structures reveal the structural transitions in alphabeta-tubulin upon GTP hydrolysis. Cell 157, 1117-1129 (2014). [PubMed: 24855948]

45. Tischfield MA \& Engle EC Distinct alpha- and beta-tubulin isotypes are required for the positioning, differentiation and survival of neurons: new support for the 'multi-tubulin' hypothesis. Biosci Rep 30, 319-330 (2010). [PubMed: 20406197]

46. Nogales E \& Zhang R Visualizing microtubule structural transitions and interactions with associated proteins. Curr Opin Struct Biol 37, 90-96 (2016). [PubMed: 26803284]

47. Atherton $\mathrm{J}$ et al. Conserved mechanisms of microtubule-stimulated ADP release, ATP binding, and force generation in transport kinesins. Elife 3, e03680 (2014). [PubMed: 25209998]

48. Pettersen EF et al. UCSF Chimera--a visualization system for exploratory research and analysis. J Comput Chem 25, 1605-1612 (2004). [PubMed: 15264254]

49. Davis IW, Murray LW, Richardson JS \& Richardson DC MOLPROBITY: structure validation and all-atom contact analysis for nucleic acids and their complexes. Nucleic Acids Res 32, W615-619 (2004). [PubMed: 15215462]

50. Altschul SF et al. Gapped BLAST and PSI-BLAST: a new generation of protein database search programs. Nucleic Acids Res 25, 3389-3402 (1997). [PubMed: 9254694]

51. Finn RD et al. HMMER web server: 2015 update. Nucleic Acids Res 43, W30-38 (2015). [PubMed: 25943547]

52. Katoh K, Misawa K, Kuma K \& Miyata T MAFFT: a novel method for rapid multiple sequence alignment based on fast Fourier transform. Nucleic Acids Res 30, 3059-3066 (2002). [PubMed: 12136088]

53. Olieric $\mathrm{N}$ et al. Automated seamless DNA co-transformation cloning with direct expression vectors applying positive or negative insert selection. BMC Biotechnol 10, 56 (2010). [PubMed: 20691119]

54. Scholz J, Besir H, Strasser C \& Suppmann S A new method to customize protein expression vectors for fast, efficient and background free parallel cloning. BMC Biotechnol 13, 12 (2013). [PubMed: 23410102]

55. Sauter NK, Grosse-Kunstleve RW \& Adams PD Robust indexing for automatic data collection. J Appl Crystallogr 37, 399-409 (2004). [PubMed: 20090869]

56. Kabsch W Automatic processing of rotation diffraction data from crystals of initially unknown symmetry and cell constants. . J. Appl. Cryst 26, 795-800 (1993).

57. McCoy AJ et al. Phaser crystallographic software. J Appl Crystallogr 40, 658-674 (2007). [PubMed: 19461840]

58. Emsley P, Lohkamp B, Scott WG \& Cowtan K Features and development of Coot. Acta Crystallogr D Biol Crystallogr 66, 486-501 (2010). [PubMed: 20383002]

59. Adams PD et al. PHENIX: building new software for automated crystallographic structure determination. Acta Crystallogr D Biol Crystallogr 58, 1948-1954 (2002). [PubMed: 12393927]

60. Vagin AA et al. REFMAC5 dictionary: organization of prior chemical knowledge and guidelines for its use. Acta Crystallogr D Biol Crystallogr 60, 2184-2195 (2004). [PubMed: 15572771]

61. DeLano WL (ed.) The PyMOL Molecular Graphics System (DeLano Scientific, Palo Alto, CA; 2002).

62. Lansbergen $\mathrm{G}$ et al. Conformational changes in CLIP-170 regulate its binding to microtubules and dynactin localisation. J Cell Biol 166, 1003-1014 (2004). [PubMed: 15381688]

63. Sindelar CV \& Downing KH The beginning of kinesin's force-generating cycle visualized at 9-A resolution. J Cell Biol 177, 377-385 (2007). [PubMed: 17470637] 
64. Sindelar CV \& Downing KH An atomic-level mechanism for activation of the kinesin molecular motors. Proc Natl Acad Sci U S A 107, 4111-4116 (2010). [PubMed: 20160108]

65. Sali A \& Blundell TL Comparative protein modelling by satisfaction of spatial restraints. J Mol Biol 234, 779-815 (1993). [PubMed: 8254673]

66. Kremer JR, Mastronarde DN \& McIntosh JR Computer visualization of three-dimensional image data using IMOD. J Struct Biol 116, 71-76 (1996). [PubMed: 8742726]

67. Farabella I et al. TEMPy: a Python library for assessment of three-dimensional electron microscopy density fits. J Appl Crystallogr 48, 1314-1323 (2015). [PubMed: 26306092]

68. Fiser A, Do RK \& Sali A Modeling of loops in protein structures. Protein Sci 9, 1753-1773 (2000). [PubMed: 11045621]

69. Emsley P \& Cowtan K Coot: model-building tools for molecular graphics. Acta Crystallogr D Biol Crystallogr 60, 2126-2132 (2004). [PubMed: 15572765]

70. Afonine PV, Headd JJ, Terwilliger TC \& Adams PD New tool: phenix.real_space_refine Computational Crystallography Newsletter 4, Part 2, 43-44 (2013).

71. Baldus M, Petkova AT, Herzfeld J \& Griffin RG Cross polarization in the tilted frame: assignment and spectral simplification in heteronuclear spin systems. Molecular Physics 95, 1197-1207 (1998).

72. Hohwy M, Rienstra CM, Jaroniec CP \& Griffin RG Fivefold symmetric homonuclear dipolar recoupling in rotating solids: Application to double quantum spectroscopy. Journal of Chemical Physics 110, 7983-7992 (1999).

73. Seidel K et al. Protein solid-state NMR resonance assignments from (C-13, C-13) correlation spectroscopy. Physical Chemistry Chemical Physics 6, 5090-5093 (2004).

74. Seidel K, Etzkorn M, Schneider R, Ader C \& Baldus M Comparative analysis of NMR chemical shift predictions for proteins in the solid phase. Solid State Nuclear Magnetic Resonance 35, 235 242 (2009) [PubMed: 19231140] 
a a human CAMSAP1 (1613 aa)

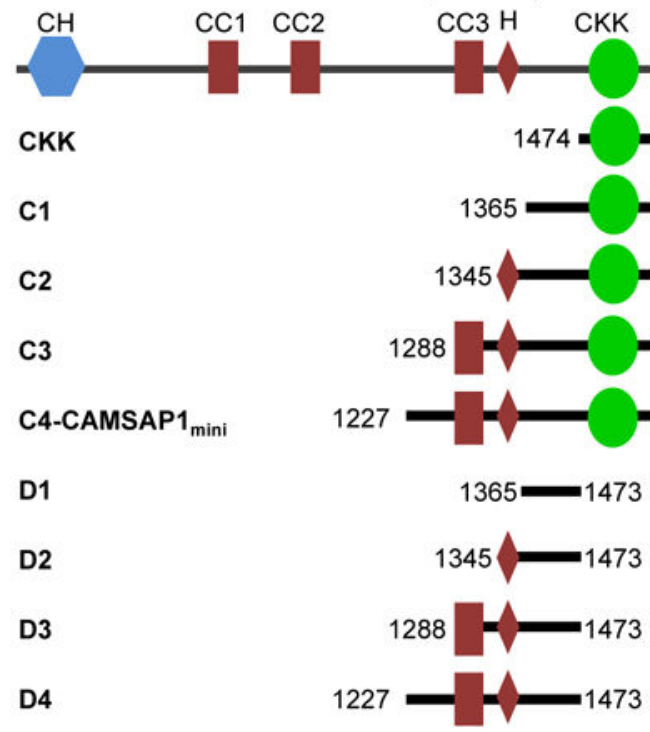

b

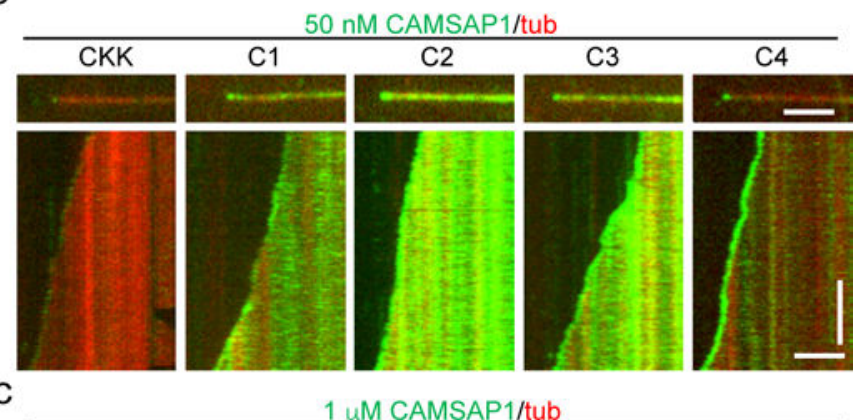

$+$
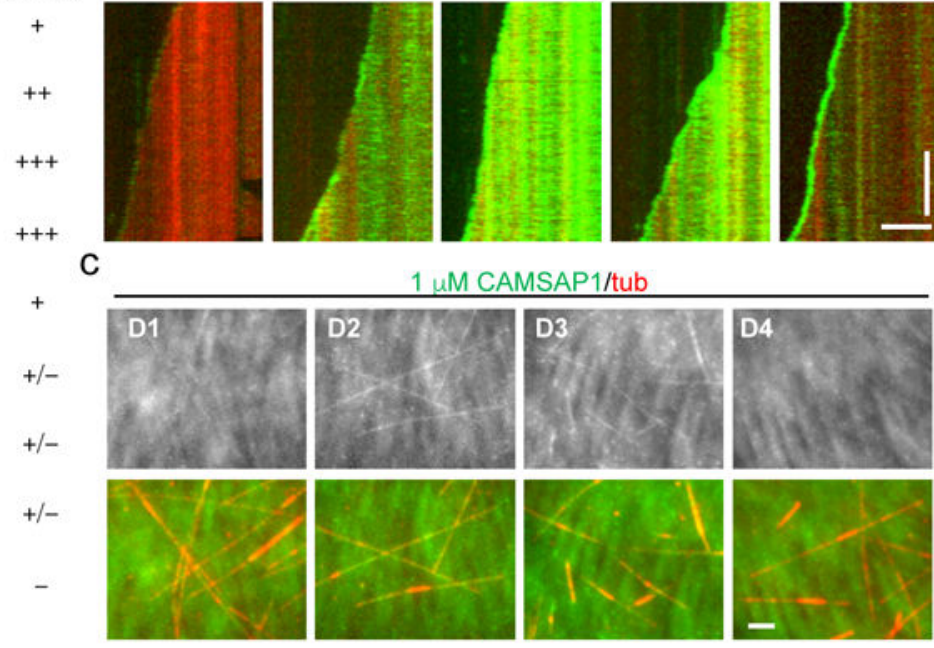

e

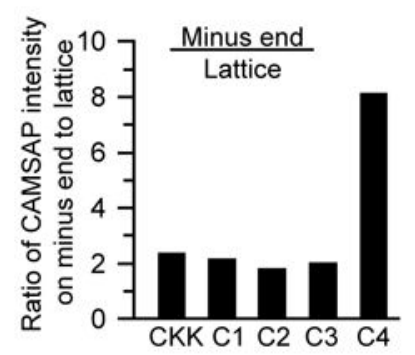

f

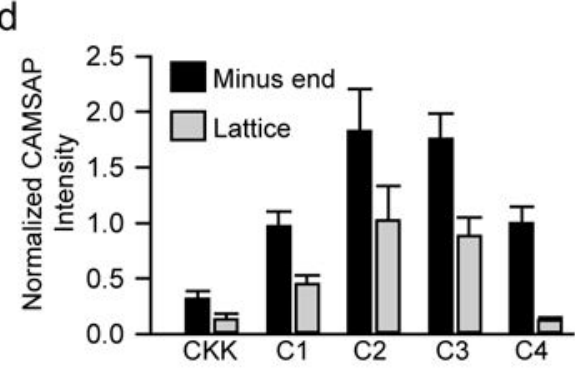

Minus end Lattice preference binding

hCAMSAP1 CKK/tub
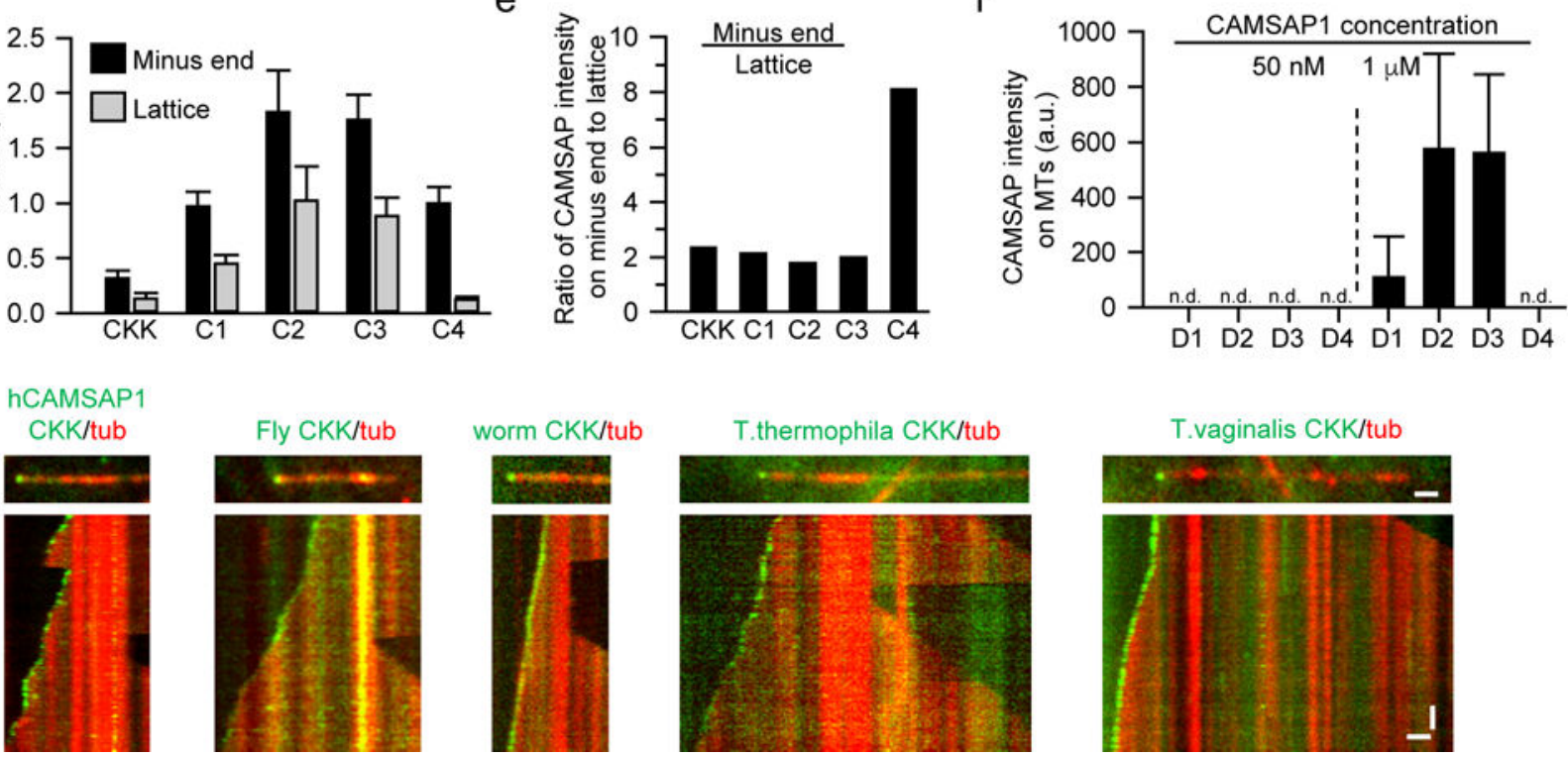

Figure 1. The CKK is a highly conserved MT minus-end tracking domain.

(a) Schematic of the CAMSAP1 domain organization and the constructs used.

(b-f) Total Internal Reflection Fluorescence Microscopy (TIRFM) images, corresponding kymographs and quantification of localization of GFP-CAMSAP1 fragments to MT minus ends and lattice. The intensity is normalized to the average minus-end intensity of the C4 fragment. Scale bars, vertical 2 min; horizontal $2 \mu \mathrm{m}$. Data represent mean \pm SD. (d, f), $\mathrm{n}=30$ MTs.

(g) TIRFM images and kymographs of GFP-tagged CKK domains from human (CAMSAP1), fly ( $D$. melanogaster), worm ( $C$. elegans), $T$. thermophila and $T$. vaginalis. Scale bars: horizontal, $1 \mu \mathrm{m}$; vertical, $30 \mathrm{sec}$. See also Supplementary Fig.1 and 2, and Supplementary Table 2 . 


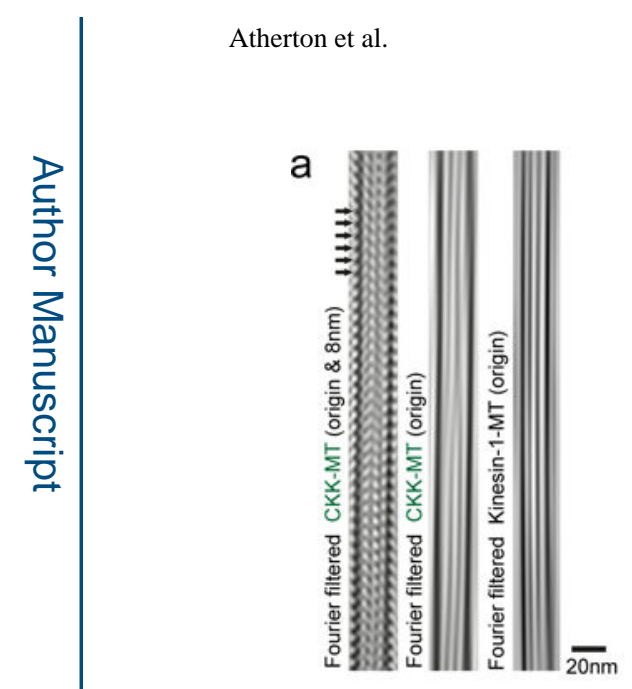

d

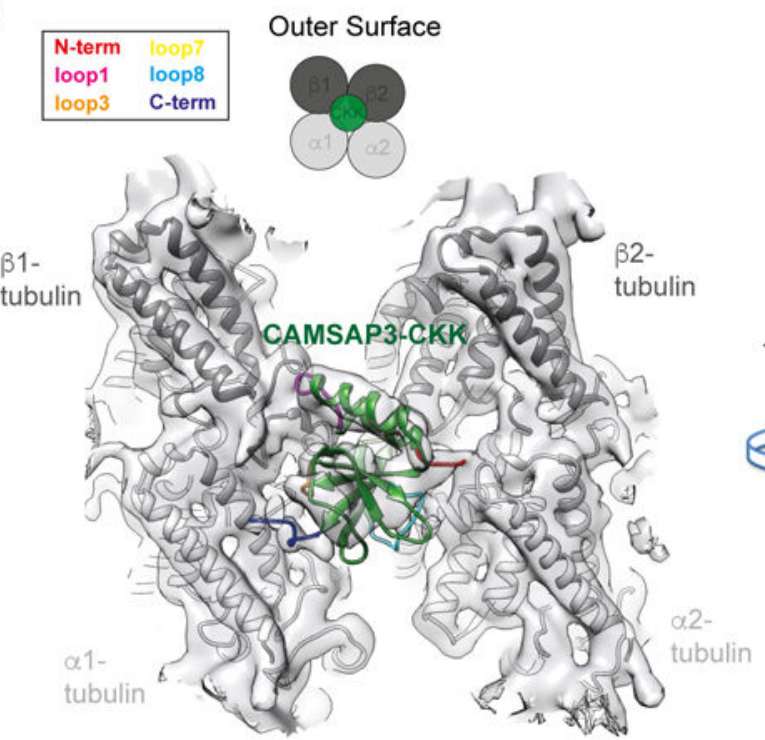

b

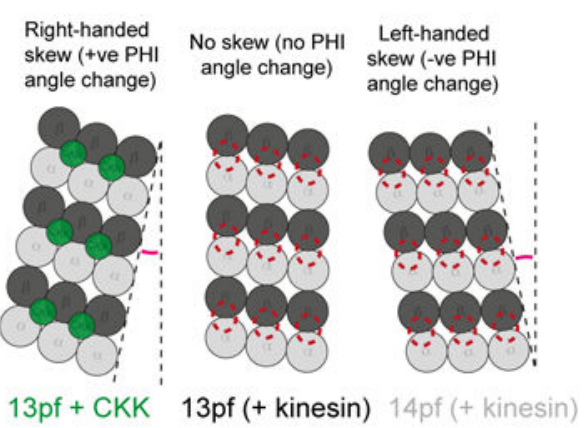

+ end

C

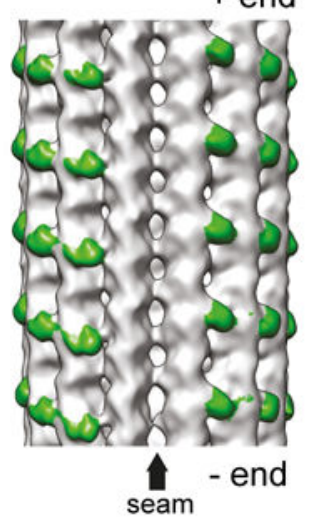

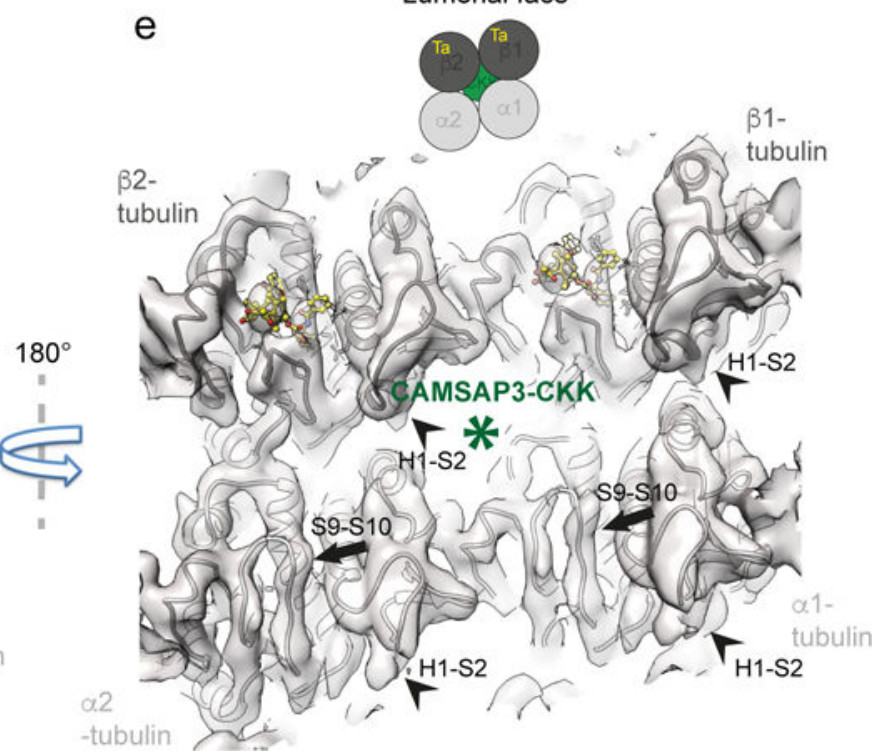

e

Lumenal face

Figure 2. The unique MT binding site of CAMSAP CKK domains.

(a) Fourier filtered images of 13pf MTs. Left, filtering of a CAMSAP3-CKK decorated 13pf

MT shows density corresponding to the CAMSAP3 CKK domain every tubulin dimer; centre, filtering highlights the MT moiré pattern and the presence of protofilament skew. Right, filtering of a kinesin-1 decorated 13pf MT highlights a comparative lack of skew. (b) Schematic of three sets of three protofilaments depicting the skew detected in the CKK 13pf MT data sets (left) compared to kinesin-bound 13pf (middle) and 14pf paclitaxelstabilized MTs (right). Skew angle size is exaggerated here for ease of depiction.

(c) The asymmetric reconstruction of the CAMSAP3-CKK decorated 13pf paclitaxelstabilized MT low-pass filtered to $15 \AA$ resolution shows extra densities (green) every 8nm corresponding to the CAMSAP3-CKK domain, which are absent at the seam (arrow). (d) The averaged reconstruction of the CAMSAP3-CKK domain viewed from the MT surface contacting two $\beta$-tubulins and two a-tubulins at the intra-dimer, inter-protofilament interface. The CKK is colored as in X-ray structure figure (see Supplementary Fig. 3) except for the $\mathrm{N}$-terminus (red) and loop1 (magenta) that are missing in our crystal structure but 
visible in our EM density. a-tubulin is shown in light grey and $\beta$-tubulin is shown in dark grey. Above, schematic.

(e) The averaged reconstruction of the MT-bound CAMSAP3-CKK domain viewed from the MT lumen showing density corresponding to paclitaxel bound to $\beta$-tubulin (yellow). Along with the distinctive appearance of the H1-S2 and S9-S10 loops (arrowheads and arrows), this allows differentiation between $\beta$ - and $\alpha$-tubulin, and thus identification of the CKK binding site at the intradimer interface. Above, schematic; Ta indicates the paclitaxel binding site. See also Supplementary Fig. 3 and 4. 

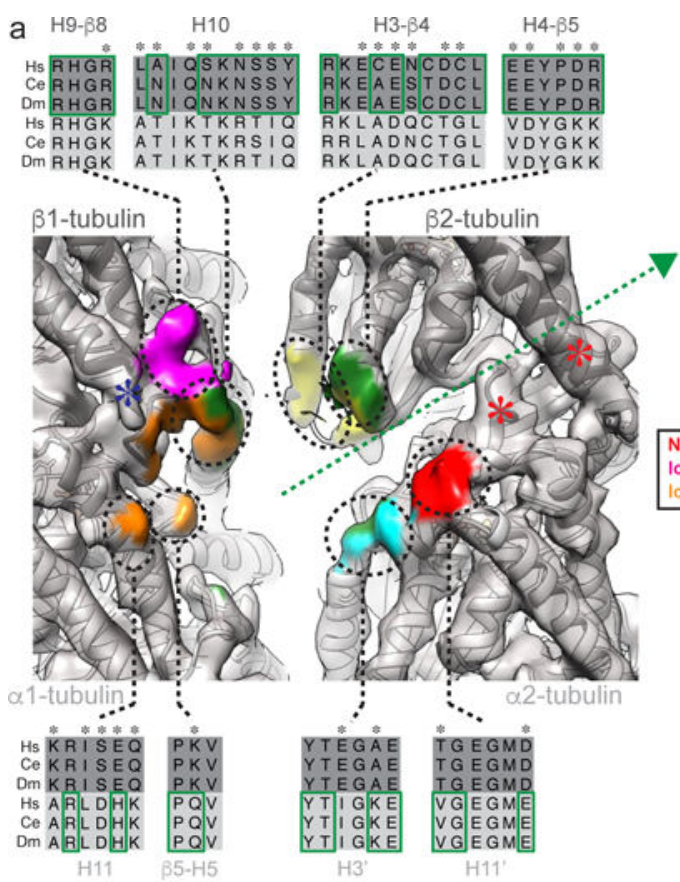

b
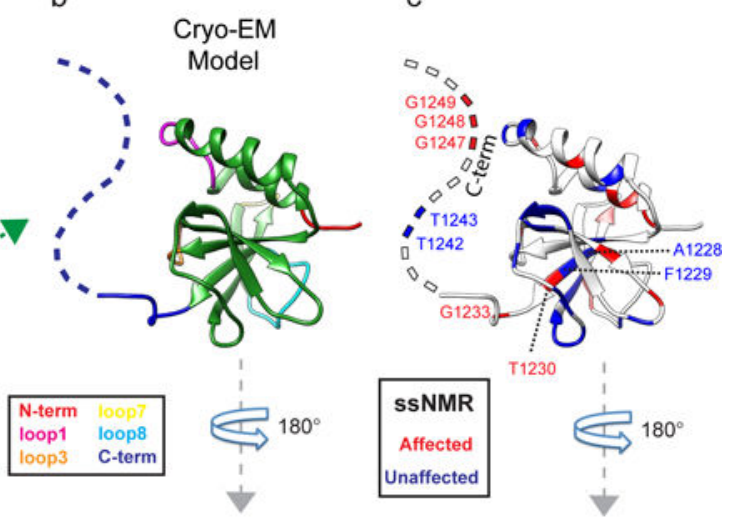

d

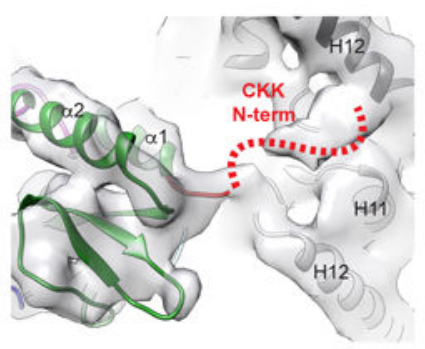

f

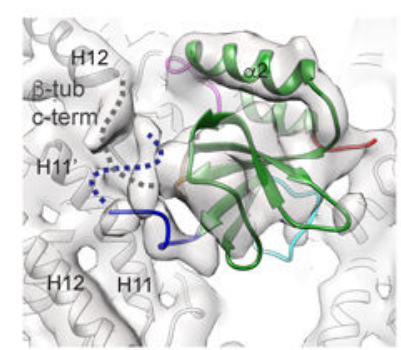

e
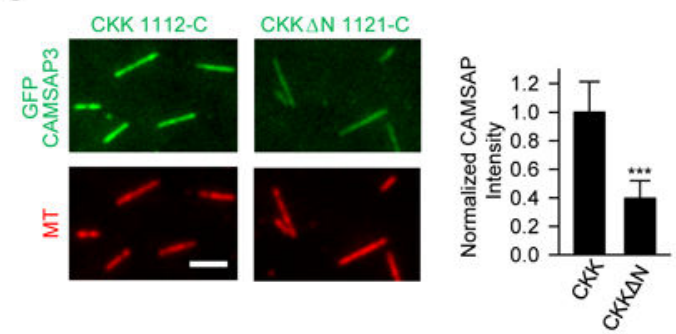

g

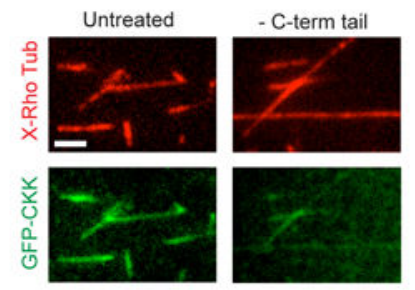

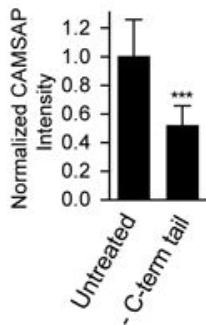

Figure 3. The interaction with four tubulin monomers is distributed across the CKK domain. (a) CKK interaction surface of the MT with cryo-EM density colored according to CKK contacts ( $<6 \AA$ distance, coloring consistent with Fig. 2d). Sequence alignments for contact regions in $\beta$-tubulin (top) and $\alpha$-tubulin (bottom) indicate sequence differences between human $\alpha$ 1a tubulin and $\beta 3$ tubulins $\left(^{*}\right)$ that could contribute to CKK binding the intra- vs the inter-dimer site. Comparison between $H$. sapiens $\beta 3$ tubulin, a 1a tubulin (most common isoforms in mammalian brain ${ }^{45}$ ), C. elegans ( $\beta 1$ tubulin, a 3 tubulin), D. melanogaster $(\beta 1$ tubulin, $\alpha$ at $84 \mathrm{~B}$ tubulin). Residues contacting the CKK are within green boundaries. 
(b) $180^{\circ}$ rotations of the CKK domain with loop coloring referring to MT contact sites in panel a.

(c) CKK views as (b), showing ssNMR data on $\left[{ }^{13} \mathrm{C},{ }^{15} \mathrm{~N}\right]$ labeled CKK decorated MTs relative to free CKK. Red: residues with significant chemical-shift or intensity changes, blue: no change. White: not analysed. The unresolved $\mathrm{N}$ - and C-termini are represented as dashed lines with each dash depicting a single residue.

(d) CAMSAP3-CKK-MT cryo-EM density at lower threshold shows the CKK N-terminus. (e) TIRFM experiments show the importance of CKK N-terminal extension in MT binding. Intensity normalized to average CKK lattice intensity. Scale bar, $2 \mu \mathrm{m}$. CKK, $\mathrm{n}=104 \mathrm{MTs}$; $\mathrm{CKK} \Delta \mathrm{N}, \mathrm{n}=118$. Data represent mean $\pm \mathrm{SD}$. ${ }^{* * *}, \mathrm{p}<0.001$, two-tailed Mann-Whitney U test. (f) CAMSAP3-CKK-MT cryo-EM density that likely corresponds to interaction between the CKK flexible C-terminus (blue dotted line) and $\beta$-tubulin C-terminus (grey dotted line), not usually seen in MT reconstructions ${ }^{46}$.

(g). X-Rhodamine labeled paclitaxel-stabilized MTs (red), either untreated or treated with subtilisin to remove their C-terminal tails, incubated with $200 \mathrm{nM}$ GFP-CAMSAP3-CKK and imaged using TIRFM. Scale bar, $4 \mu \mathrm{m}$. The intensity of MT labelling, normalized to wild type, was quantified; $\mathrm{n}=100$ MTs. Data represent mean \pm SD. ${ }^{* * *}, \mathrm{p}<0.001$, two-tailed Mann-Whitney U test.

See also Supplementary Fig. 5 and Supplementary Table 2. 


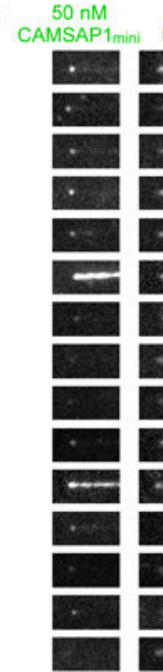

b

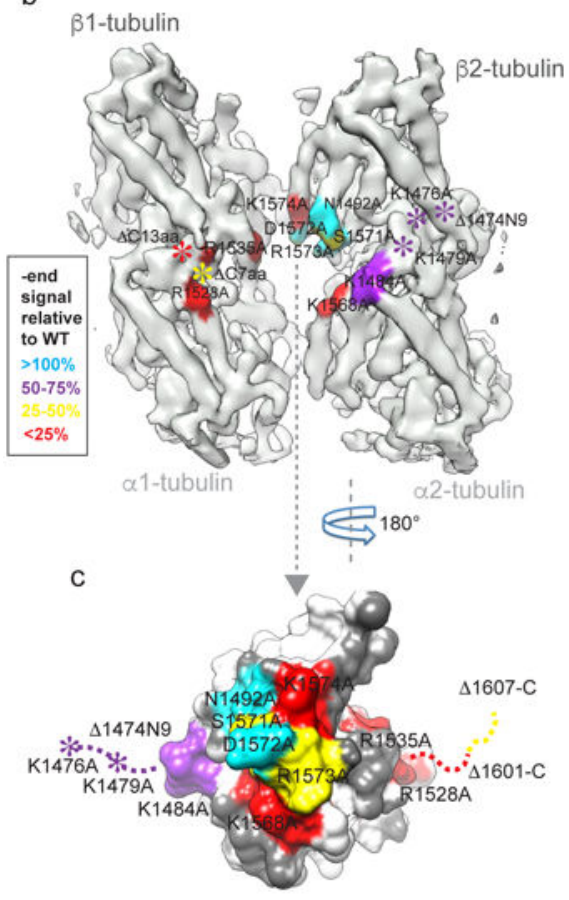

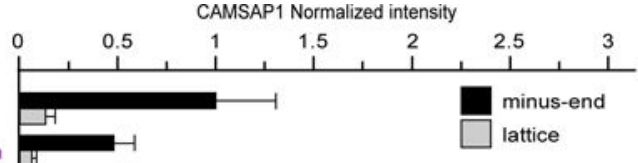

H

$\begin{array}{ll}\triangle 1112 \mathrm{~N} 9 & \mathrm{~N} \text { extension } \\ \text { R1114A } & \mathrm{N} \text { extension }\end{array}$

K1117A N extension

K1122A N extension

N1130A Helix $\alpha 1$

R1166A Loop 3

R1173A B2 sheet

K1206A

S1209A

D1210A

R1211A

K1212A

$\triangle 1245-\mathrm{C}$

$\Delta 1239-\mathrm{C} C$ extension

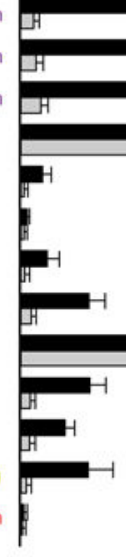

d
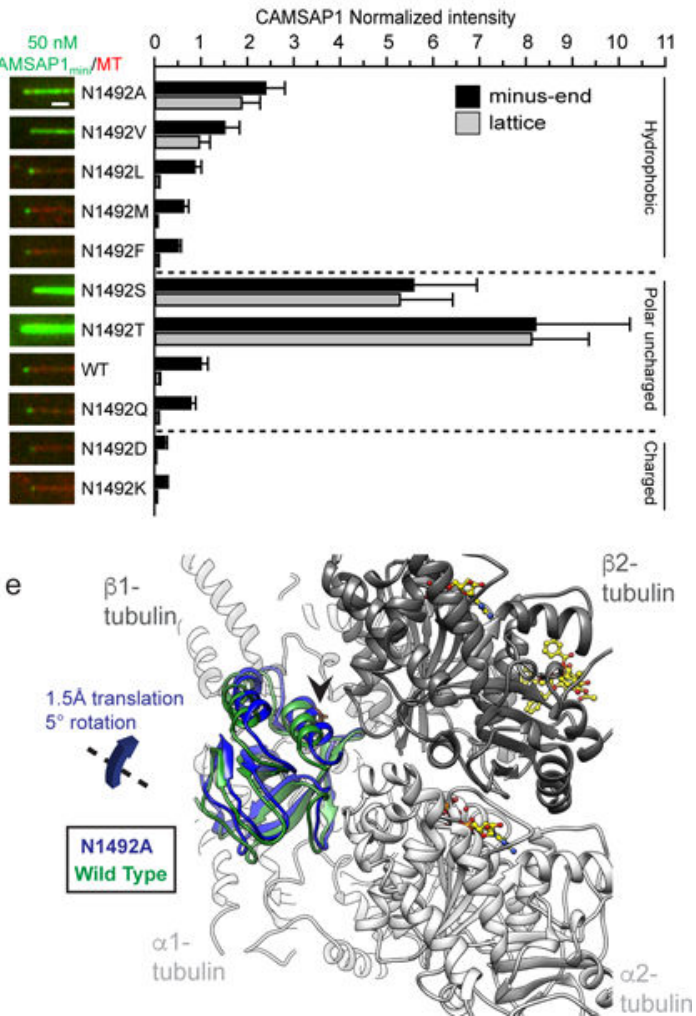

Figure 4. Validation of CKK-MT contact sites using in vitro assays and structure of a mutant CKK bound to MTs.

(a) Left, TIRFM images of GFP-CAMSAP1 $1_{\text {mini }}$ wild type and mutants binding to the minus ends of dynamic MTs. The corresponding residues in CAMSAP3 and their location are indicated. Scale bar, $1 \mu \mathrm{m}$. Right, quantification of GFP-CAMSAP $1_{\text {mini }}$ intensities at MT minus ends and on MT lattice. The intensity is normalized to the average minus-end intensity of wild type. $\mathrm{n}$ ranged from 17 to $87 \mathrm{MTs}$; individual data points are provided in Supplementary Table 2. Data represent mean \pm SD. 
(b) View of the CKK interaction surface of the MT cryo-EM density with mutated CKK residues mapped ( $<8 \AA$ distance) and colored according to the $\%$ change in minus-end fluorescence signal relative to wild type of corresponding mutants (panel a).

(c) Surface representation of the tubulin-interacting face of the CAMSAP1 CKK domain. Mutated CKK residues are colored according to the \% change in minus-end fluorescence signal of corresponding mutants relative to wild type in our TIRF assays (as in panel a). (d) Left, TIRFM images of GFP-CAMSAP1 $1_{\text {mini }}$ N1492 mutants; Scale bar, $1 \mu \mathrm{m}$. Right, quantification of GFP-CAMSAP $1_{\text {mini }}$ intensities at MT minus ends and on MT lattice. The intensity is normalized to the average minus-end intensity of wild type. $n=30$ MTs. Scale bar, $1 \mu \mathrm{m}$

(e) The N1492A CAMSAP1 CKK binds at the intra-dimer, inter-protofilament MT binding site but in a subtly different orientation compared to wild type. Ribbon representation comparing the position of N1492A CAMSAP1 CKK with wild type CAMSAP1 CK relative to the tubulin-binding surface. N1492A CAMSAP1 CKK (shown in blue) is rotated $5^{\circ}$ (around the indicated axis) and translated $1.9 \AA$ into the inter-protofilament binding site compared to wild type CAMSAP1 CKK (green). Arrowhead depicts position of N1492. See also Supplementary Fig. 7 and 8, and Supplementary Table 2. 
a
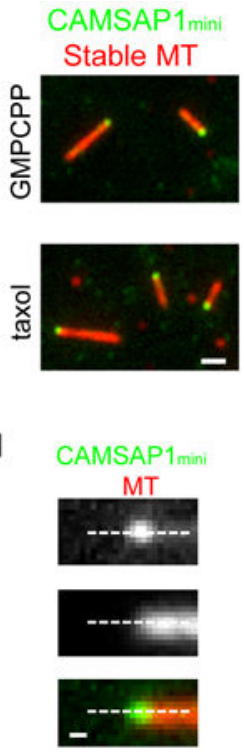

b
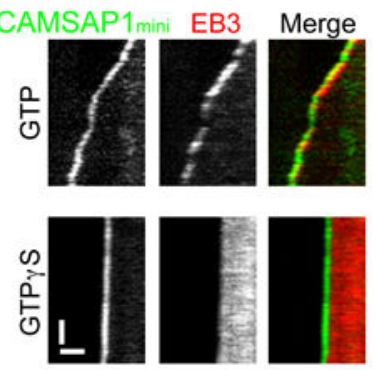
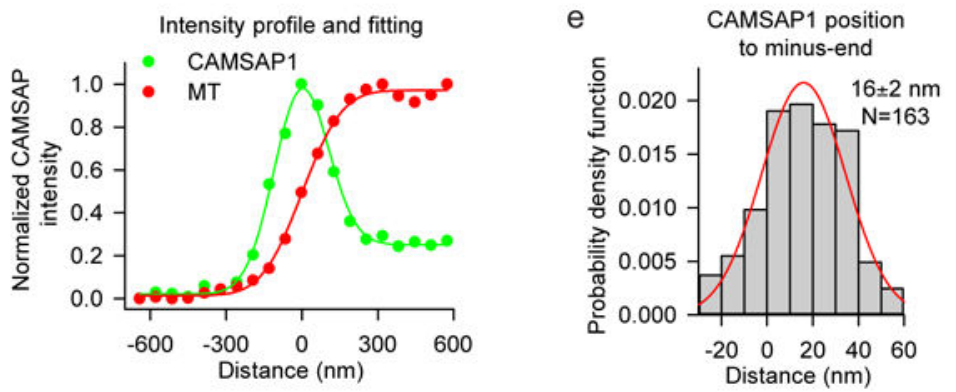

f

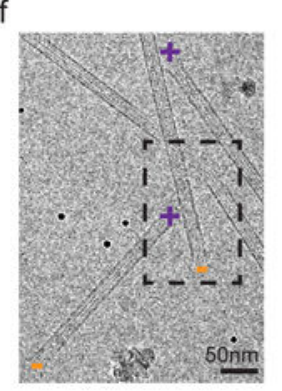

g
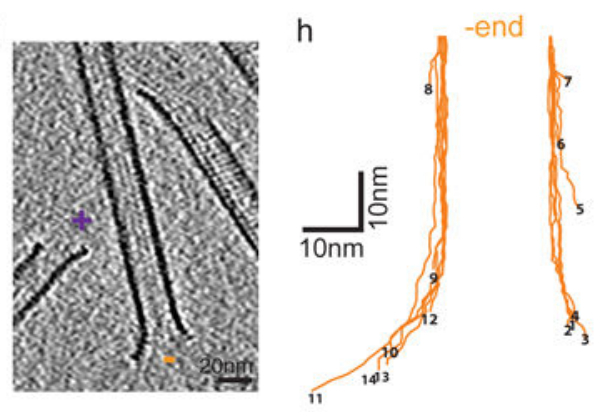

i

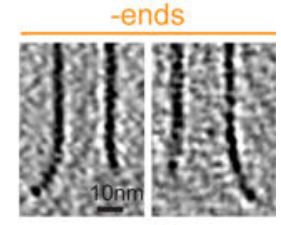

+ends

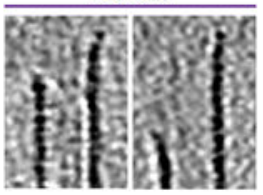

$\mathrm{k}$

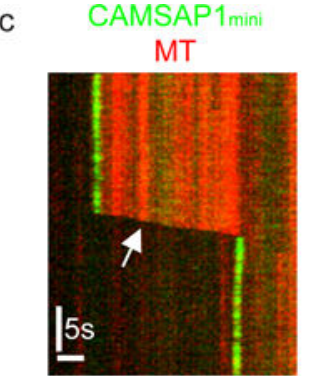

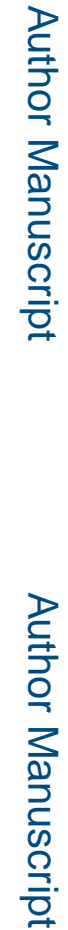

j

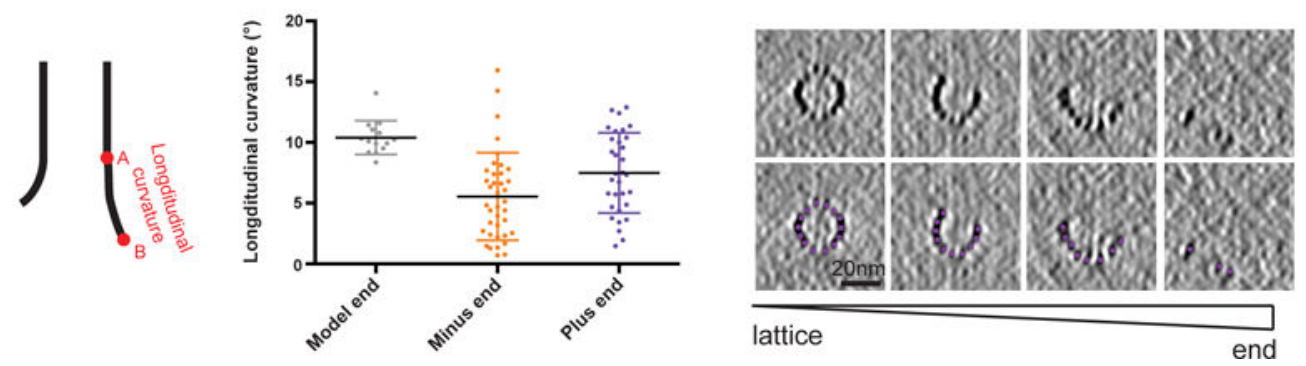

Figure 5. Examining CKK's preferred tubulin conformation.

(a) TIRFM images of the minus-end localization of GFP-CAMSAP $1_{\text {mini }}$ on GMPCPP or taxol-stabilized MTs. Scale bar, $1 \mu \mathrm{m}$.

(b) Kymographs showing that GFP-CAMSAP $1_{\text {mini }}$ tracks growing MT minus ends whether MTs are polymerized in the presence of GTP or GTP $\gamma \mathrm{S}$, while mCherry-EB3 decorates the whole lattice of GTP $\gamma \mathrm{S}$ MTs. Scale bars: horizontal, $1 \mu \mathrm{m}$; vertical, $30 \mathrm{sec}$.

(c) Kymograph showing that GFP-CAMSAP $1_{\text {mini }}$ tracks a growing but not a depolymerizing MT minus end (white arrow). Scale bars: horizontal, $1 \mu \mathrm{m}$; vertical, $5 \mathrm{sec}$. 
(d) A TIRFM image of Rhodamine-labeled stable GMPCPP MT and CAMSAP1 $1_{\text {mini-GFP. }}$ Normalized one-dimensional intensity profiles of CAMSAP 1 mini-GFP and MT and corresponding fitting of point spread function-convoluted models (see Methods for details). To reduce the error introduced by the flexible linker between CKK and GFP, GFP was inserted at the CKK C-terminus. Scale bar, $200 \mathrm{~nm}$.

(e) Distribution of the position of CAMSAP1 ${ }_{\text {mini- }}$ GFP relative to the MT minus end (mean \pm SEM). N=163 MTs.

(f) Projection image from a cryo-ET tilt-series showing in vitro GMPCPP-MTs including several ends. The moiré patterns were used to determine MT polarity (see Supplementary Fig. 9). The black dots are gold fiducials for tilt series alignment.

(g) Longitudinal slice through MTs in the tomographic volume corresponding to the boxed region in panel $\mathrm{f}$.

(h) 2D graphical representation of an exemplar minus end, plotting 3D pf trajectories. In this $14 \mathrm{pf}$ MT, 7 pfs are plotted on either side, numbered around the circumference (pf14 is adjacent to pf1).

(i) Sagittal slices through tomographic volumes showing a range of curvatures and lengths at both ends. Some pfs terminate in the lattice before curvature is observed.

(j) Longitudinal curvature of ends. Only curved end regions $>$ one dimer long are plotted. Inset; schematic of longitudinal curvature A-B. Model End (Supplementary Fig. 9c), n=14 protofilaments; Plus End, $n=32$ protofilaments ( 5 MTs), Minus End, $n=38$ protofilaments ( 5 MTs). Data represent mean \pm SD. Minus End vs Plus End not significant; model vs data, statistically significant $(\mathrm{p}<0.01)$ Kolmogorov-Smirnov test.

(k) Series of transverse sections through a MT from lattice to end. Lower panels show traced pf positions.

See also Supplementary Fig. 9 and Supplementary Table 2. 


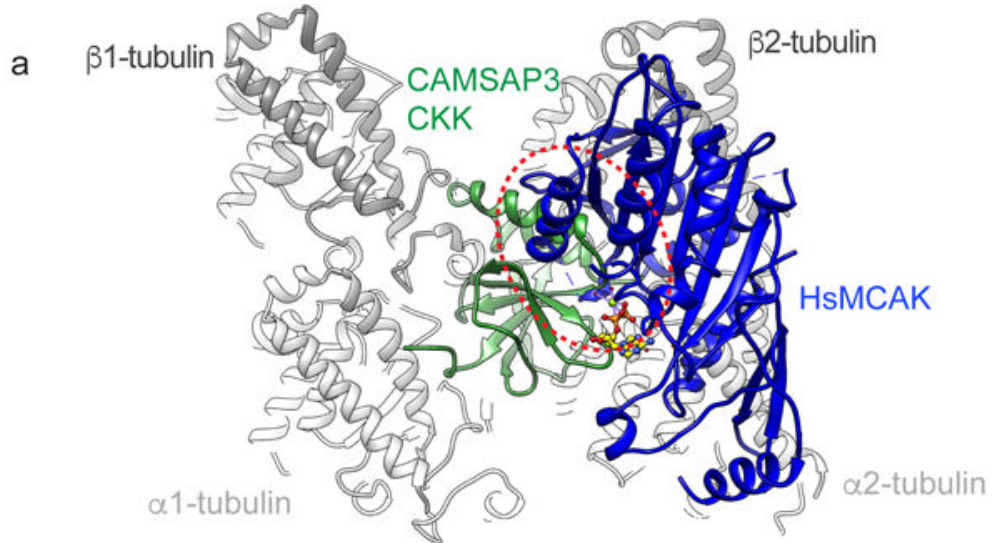

b

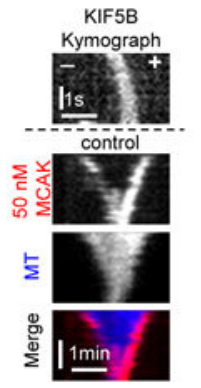

C
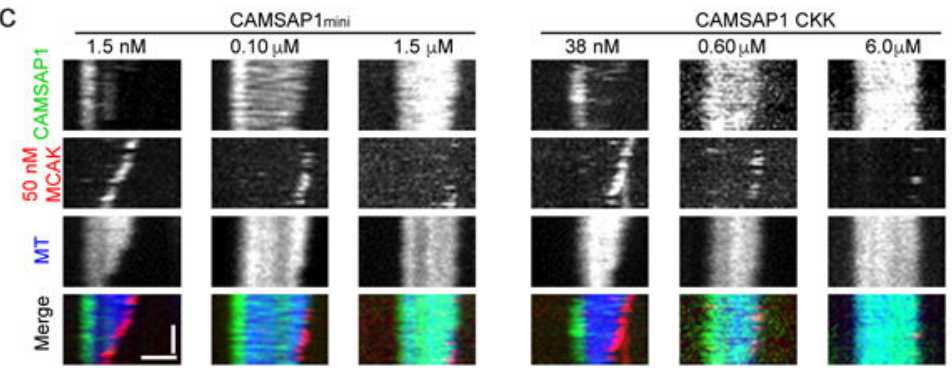

d

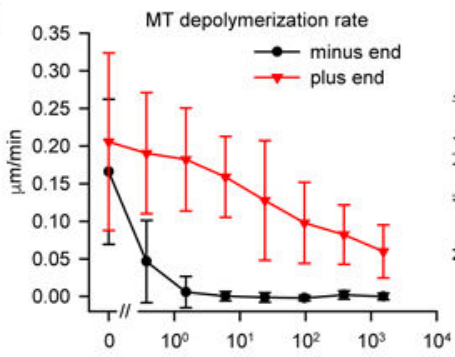

e
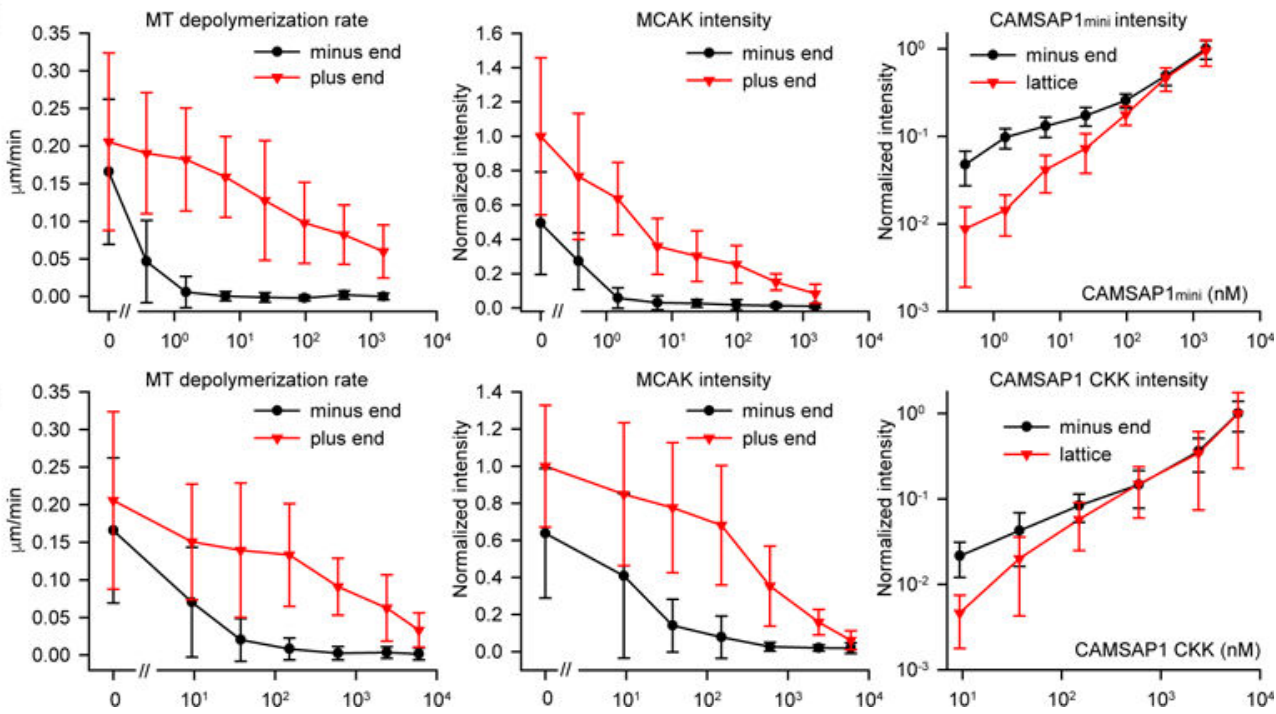

Figure 6. CAMSAP CKKs protect MT minus ends from MCAK-induced depolymerization via steric inhibition.

(a) A MT tubulin dimer-pair bound to CAMSAP3-CKK (green) is shown with the expected position of an MD of human MCAK (in complex with ADP; PDB ID 4UBF) by alignment with MT-bound kinesin- $1^{47}$ in Chimera ${ }^{48}$.

b) Kymographs of MT depolymerization assay with GMPCPP-stabilized MTs (blue) and GFP-MCAK (red). MT polarity was determined based on the movement of the SNAP- 
Alexa647-tagged plus-end directed motor kinesin-1 KIF5B (green, residues 1-560). Scale bars: horizontal, $1 \mu \mathrm{m}$; vertical, upper panel, $1 \mathrm{sec}$, lower panels, $1 \mathrm{~min}$.

c) Kymographs of MT depolymerization assays with GMPCPP-stabilized MTs (blue), GFPMCAK (red) and different concentrations of SNAP-Alexa 647 CAMSAP $1_{\text {mini }}$ or CKK (green). Scale bars: horizontal, $1 \mu \mathrm{m}$; vertical, 1 min. In panels (b) and (c), MT minus (-) ends are shown on the left and plus (+) ends on the right.

d-e) Quantification of MT depolymerization rate, MCAK intensity and CAMSAP1 intensity at different concentrations of CAMSAP $1_{\text {mini }}$ (d) or CKK (e). $n$ ranged from 17 to 31 MTs; individual data points are provided in Supplementary Table 2. Data represent mean \pm SD. See also Supplementary Table 2. 


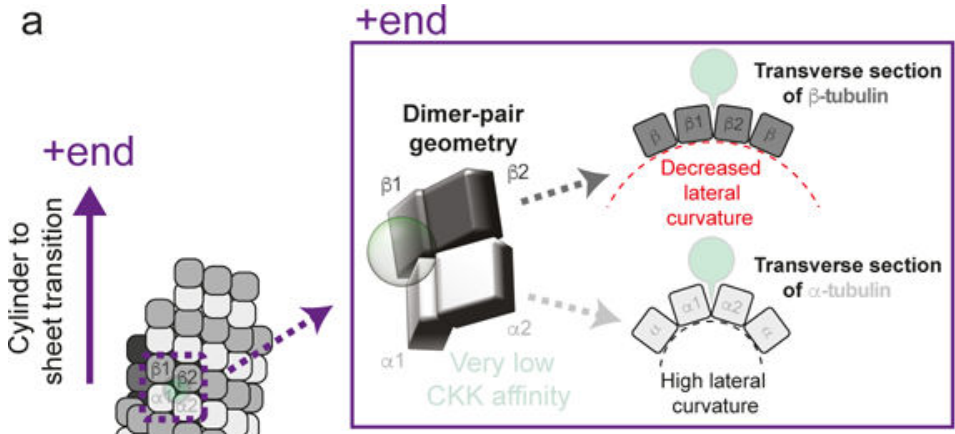

Lattice

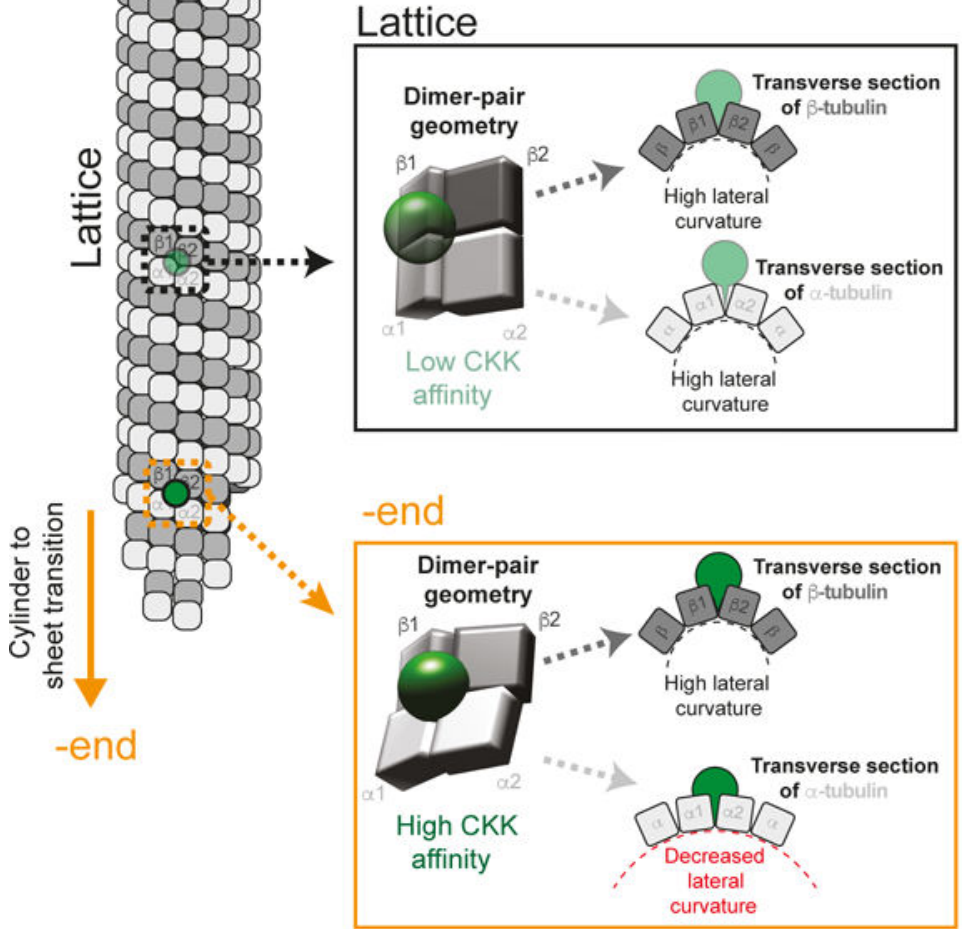

b

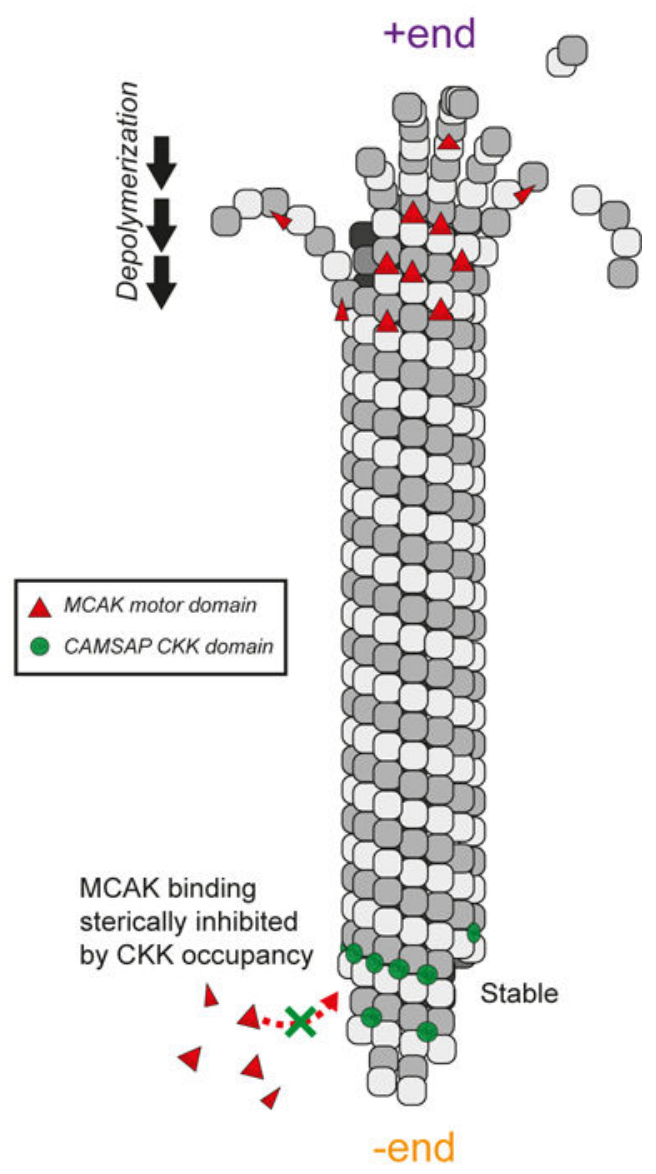

Figure 7. Proposed mechanisms of MT minus end binding and protection from MCAK-induced depolymerization by the CKK domain.

(a) Towards the ends of stable or growing MTs there is a transition from the regular lattice to sheet-like regions, with increasing longitudinal curvature and decreasing lateral curvature. Protofilaments retain lateral connectivity, and thus inter-protofilament CKK binding sites are preserved. Given the polar nature of MTs, these lattice-sheet transitions create unique conformations of B-lattice tubulin dimer-pairs at either end of the MT: at the minus end, $a-$ tubulins in dimer-pairs are more flattened compared to $\beta$-tubulins, with the opposite being true at the plus end. In this way, we propose that the unique dimer-pair conformation at the minus end favors CKK binding when compared to dimer pair conformations in the lattice or at the plus end.

(b) Model of minus end (-end) specific protection from MCAK-induced depolymerization by the CKK domains. CKKs (green circles) bind specifically to a curved minus end region, preventing the association of MCAK motor domains (red triangles) to the same region via 
steric inhibition. As the CKKs do not bind to the corresponding region of the plus end (+end), MCAK is free to bind and depolymerise from the plus end. 


\section{Table 1}

Data collection and refinement statistics (molecular replacement)

\begin{tabular}{|c|c|}
\hline & $\begin{array}{c}\text { CKK3 } \\
(5 \mathrm{LZN})\end{array}$ \\
\hline \multicolumn{2}{|l|}{ Data collection } \\
\hline Space group & I422 \\
\hline \multicolumn{2}{|l|}{ Cell dimensions } \\
\hline$a, b, c(\AA)$ & $96.4,96.4,63.3$ \\
\hline$a, \beta, \gamma\left(^{\circ}\right)$ & $90.0,90.0,90.0$ \\
\hline Resolution $(\AA)$ & $\begin{array}{l}68.1-1.4(1.45- \\
1.4)\end{array}$ \\
\hline $\begin{array}{l}R_{\text {meas }}(\%) \\
R_{\text {pim }}(\%)\end{array}$ & $\begin{array}{c}6.8(475.0) \\
1.3(99.2)\end{array}$ \\
\hline$I / \sigma(I)$ & $23.5(0.8)$ \\
\hline$C C_{1 / 2}$ & $100(52.2)$ \\
\hline Completeness (\%) & $100(99.9)$ \\
\hline Redundancy & $25.5(22.4)$ \\
\hline \multicolumn{2}{|l|}{ Refinement } \\
\hline Resolution (̊̊) & $68.1-1.4$ \\
\hline No. reflections & 29557 \\
\hline$R_{\text {work }} / R_{\text {free }}$ & $16.2 / 19.2$ \\
\hline \multicolumn{2}{|l|}{ No. atoms } \\
\hline Protein & 897 \\
\hline Water & 67 \\
\hline \multicolumn{2}{|l|}{$B$ factors } \\
\hline Protein & 46.0 \\
\hline Water & 45.5 \\
\hline \multicolumn{2}{|l|}{ R.m.s. deviations } \\
\hline Bond lengths $(\AA)$ & 0.01 \\
\hline Bond angles $\left({ }^{\circ}\right)$ & 1.33 \\
\hline
\end{tabular}

Data were collected from one crystal 
Table 2.

Cryo-EM data collection, refinement and validation statistics

\begin{tabular}{|c|c|c|c|}
\hline & $\begin{array}{c}\text { CAMSAP3 CKK } \\
\text { taxol-GDP } \\
\text { 13pf MTs } \\
\text { (EMD-4154, 5M50) }\end{array}$ & $\begin{array}{c}\text { CAMSAP1 CKK } \\
\text { taxol-GDP } \\
\text { 13pf MTs } \\
\text { (EMD-4156, 5M54) }\end{array}$ & $\begin{array}{c}\text { CAMSAP1 N1492A } \\
\text { CKK taxol- GDP } \\
\text { 13pf MTs } \\
\text { (EMD-3444, 5M5C) }\end{array}$ \\
\hline \multicolumn{4}{|l|}{$\begin{array}{l}\text { Data collection and } \\
\text { processing }\end{array}$} \\
\hline Magnification & 41,720 & 41,477 & 35,971 \\
\hline Voltage (kV) & $300 \mathrm{Kv}$ & $200 \mathrm{Kv}$ & $300 \mathrm{Kv}$ \\
\hline Electron exposure $\left(\mathrm{e}-/ \AA^{2}\right)$ & 25 & 20 & 25 \\
\hline Defocus range $(\mu \mathrm{m})$ & -0.4 to $-3.5 \mu \mathrm{m}$ & -0.4 to $-3.5 \mu \mathrm{m}$ & -0.4 to $-3.5 \mu \mathrm{m}$ \\
\hline Pixel size $(\AA)$ & $1.534 \AA$ & $1.543 \AA$ & $1.39 \AA$ \\
\hline Symmetry imposed ${ }^{*}$ & Pseudo-helical & Pseudo-helical & Pseudo-helical \\
\hline Initial particle images (no.) & 66,453 & 34,122 & 36,027 \\
\hline Final particle images (no.) & 6,530 & 5,954 & 4,144 \\
\hline $\begin{array}{l}\text { Map resolution }(\AA) \\
\text { FSC threshold }\end{array}$ & $\begin{array}{c}5.3 \AA \\
\text { FSCtrue } 0.143\end{array}$ & $\begin{array}{c}8 \AA \\
\text { FSCtrue } 0.143\end{array}$ & $\begin{array}{c}4.8 \AA \\
\text { FSCtrue } 0.143\end{array}$ \\
\hline Map resolution range $(\AA)$ & $5-6.5 \AA$ & $7.5-8.5 \AA$ & $4.5-6 \AA$ \\
\hline \multicolumn{4}{|l|}{ Refinement } \\
\hline Initial models used & 5LZN, 1UGJ, 3J6G & 5LZN, 1UGJ, 3J6G & 5LZN, 1UGJ, 3J6G \\
\hline Refinement resolution $(\AA)$ & 6.5 & 8 & 5.5 \\
\hline $\mathrm{FSC}_{\text {average }}$ & 0.81 & 0.91 & 0.83 \\
\hline Map sharpening $B$ factor $\left(\AA^{2}\right)$ & -200 & -350 & -120 \\
\hline \multicolumn{4}{|l|}{ Model composition } \\
\hline Nonhydrogen atoms & 14,600 & 14,605 & 14,608 \\
\hline Protein residues & 1826 & 1825 & 1826 \\
\hline Ligands & 6 & 6 & 6 \\
\hline \multicolumn{4}{|l|}{ R.m.s. deviations } \\
\hline Bond lengths $(\AA)$ & 0 & 0 & 0 \\
\hline Bond angles $\left({ }^{\circ}\right)$ & 0.78 & 0.72 & 0.79 \\
\hline \multicolumn{4}{|l|}{ Validation ${ }^{\#}$} \\
\hline MolProbity score & 1.70 & 1.61 & 1.70 \\
\hline Clashscore & 8.94 & 7.15 & 7.4 \\
\hline Poor rotamers (\%) & 0 & 0 & 0.06 \\
\hline \multicolumn{4}{|l|}{ Ramachandran plot } \\
\hline Favored (\%) & 96.58 & 96.58 & 95.75 \\
\hline Allowed (\%) & 100 & 100 & 100 \\
\hline Disallowed (\%) & 0 & 0 & 0 \\
\hline
\end{tabular}


${ }^{\#}$ As defined by the MolProbity 49 validation server. 